\title{
A Novel Portfolio Based on Interval-Valued Intuitionistic Fuzzy AHP with Improved Combination Weight Method and New Score Function
}

\section{Xue Deng}

South China University of Technology

Fengting Geng ( $\nabla$ ftGengscut@163.com )

South China University of Technology

Jianxin Yang

South China University of Technology

\section{Research Article}

Keywords: Interval-valued intuitionistic fuzzy AHP, Portfolio, Score function, Combination weighted method

Posted Date: December 6th, 2021

DOI: https://doi.org/10.21203/rs.3.rs-1130173/v1

License: (c) (i) This work is licensed under a Creative Commons Attribution 4.0 International License. Read Full License 


\title{
A Novel Portfolio Based on Interval-Valued Intuitionistic Fuzzy AHP with Improved Combination Weight Method and New Score Function
}

\begin{abstract}
Xue Deng, Fengting Geng*, Jianxin Yang
Abstract:

The classical Analytic Hierarchy Process (AHP) requires an exact value to compare the relative importance of two attributes, but experts often can not obtain an accurate assessment of every attribute in the decision-making process, there are always some uncertainty and hesitation. Compared with classical AHP, our new defined interval-valued intuitionistic fuzzy AHP has accurately descripted the vagueness and uncertainty. In decision matrix, the real numbers are substituted by fuzzy numbers. In addition, each expert will make different evaluations according to different experiences for each attribute in the subjective weighting method, which neglects objective factors and then generates some deviations in some cases. This paper provides two ways to make up for this disadvantage. On the one hand, by combining the interval-valued intuitionistic fuzzy AHP with entropy weight, an improved combination weighting method is proposed, which can overcome the limitations of unilateral weighted method only considering the objective or subjective factors. On the other hand, a new score function is presented by adjusting the parameters, which can overcome the invalidity of some existing score functions. In theory, some theorems and properties for the new score functions are given with strictly mathematical proof to validate its rationality and effectiveness. In application, a novel fuzzy portfolio is proposed based on the improved combination weighted method and new score function. A numerical example shows that these results of our new score function are consistent with those of most existing score functions, which verifies that our model is feasible and effective.
\end{abstract}

Key words: Interval-valued intuitionistic fuzzy AHP; Portfolio; Score function; Combination weighted method.

\section{Introduction}

The portfolio theory was first proposed by Markowitz [1] in 1952. He used the average rate of historical return to measure the expected return level of investments and the variance of the rate of return to measure the investment risk. On this basis, the mean-variance model was established to explain how to achieve the best balance between returns and risks through the selection of securities portfolios. The central problem of portfolio theory is how to choose the combination of return and risk in the decision of securities investment. In this way, the expected benefits can be maximized at a given level of expected risk, or the expected risks can be minimized at a given level of expected return. Makovitz assumed that the income distribution was symmetric and used the variance to depict the risk. But in actual cases, variance is not always accurate to describe the risk, and the distribution of income is not necessarily symmetric. So, many scholars put forward their own different views on this. Hogan and Warren [2], Harlow [3] proposed that the lower half variance can depict the risk more accurately and discussed he mean half variance model. Konno and Suzuki [4] studied the mean variance-skewness model which is valuable in the case of asymmetric return distribution. Konno and Yamazaki [5] used expected absolute deviation to describe risks and established a linear programming model for portfolio selection, which is called mean absolute deviation model. Athayde and Flores [6] considered the asset allocation under the condition of asymmetric distribution. Jondeau and Rockinger [7] considered the non-normal distribution and time-varying characteristics of the rate of return. In their paper, Taylor expansion was performed on the final expected income and the first four high order

\footnotetext{
* Corresponding author. Present address: School of Mathematics, South China University of Technology, Guangzhou, China, 510640 .

E-mail address: ftGengscut@163.com (F.T. Geng).
} 
moments are taken, and the first order condition was used to optimize the asset allocation. Li [8] constructed an asymmetry robust mean absolute deviation (ARMAD) model that takes the asymmetry distribution of returns into consideration. Deng and Yuan [32] constructed a hybrid multi-objective portfolio model which considers fuzzy return status, systematic risk, non-systematic risk and entropy.

The concept of entropy originating from thermodynamics reflects the degree of chaos in a system. The smaller the corresponding entropy value of the system, the more stable the system. Zadeh [9] put forward the concept of fuzzy entropy for the first time in 1965. Then many scholars had offered different definitions of interval intuitionistic fuzzy entropy. In the case of attributes was completely unknown, most scholars used entropy weight method to determine weights. Burillo and Bustince [30] proposed the notions of entropy of to measure the degree of intuitionism of interval-valued intuitionistic fuzzy sets and intuitionistic fuzzy sets. Szimidt and Kacprzyk [31] proposed a non-probabilistic-type entropy measure with a geometric interpretation of intuitionistic fuzzy sets. Deng and Liu [33] used improved entropy method to calculated the weight of each indicator in order to conduct quantitative analysis on 20 indicator variables which can be divided into four digital economic types in Guangdong Province from 2015 to 2018.

Analytic hierarchy process [10] is a multi-criteria decision-making method combining qualitative and quantitative analysis, which is practical in the case of complex target structure and lack of necessary data. Because the fuzziness of expert judgment is not considered when evaluating the weight distribution of various factors, Atanassov [11,12] successively proposed the intuitionistic fuzzy set and the interval-valued intuitionistic fuzzy set to effectively solve the double fuzzy situation in life. Sadiq [13] applied the intuitionistic fuzzy set to the AHP and then construct the intuitionistic fuzzy AHP method.

The sort function is a mean to measure the intuitionistic fuzzy number. In order to compare the advantages and disadvantages of two interval-valued intuitionistic fuzzy numbers, $\mathrm{Xu}$ [14] defined the score function and the accurate function of interval-valued intuitionistic fuzzy numbers as the sort function, and gave the corresponding sorting rules. However, Xu's ordering rules are invalid for some interval-valued intuitionistic fuzzy numbers. Therefore, many scholars put forward new sort functions from different perspectives. Ye [23] proposed the accurate function from the perspective of hesitation in 2009. Nayagam and Sivaraman et al. [24, 25], Gao [26], Kang [27] and Wang [28, 29] proposed some different sort functions. But there are still cases of sorting failure.

The inherent statistical rules and authoritative values among index data should be considered when assigning weights to indexes. Many scholars have studied different combination weighting methods to make up for the limitations of a single weighting method. Wang [16] used combination weighting method and the fuzzy multi-criteria model to select the optimal cool storage system for air conditioning. In the evaluation process, the optimal weighting method combined the subjective knowledge of decision-maker and the objectivity of numerical data to obtain the comprehensive weights of criteria and avoided the subjective one-sidedness of weights. To get the subjective and objective weights, Yi [17] used fuzzy analytic hierarchy process method and improved criteria importance through inter-criteria correlation, and he applied the least square method to obtain the combined weights, which reduced the influence of artificial experience. Hu [18] established a credit evaluation model based on the combination weighting method, considering the information volume, volatility, and difference of the road transportation enterprises data and using normalized constraints of maximum variance to determine the combination weights. The model fully considered the degree of difference between the indicators and made up for the deviation of the single weighting method. Tan [19] used an improved analytic hierarchy process AHP and the entropy method to make the suitability evaluation of underground space. The method ensured rationality of the evaluation results to the greatest extent, thereby providing a certain guiding significance for the development of underground space. Wu [20] used the coefficient of variation method and entropy weight method to 
determine the combined weight of the evaluation indicators, and realized the optimization of the green building programs in the South Sichuan Economic Zone.

As one kind of decision problem, portfolio selection also needs to take the subjective knowledge of investor into account. In addition, to better describe the uncertainty of financial market, using interval-valued fuzzy can obtain more detailed information of securities. For this purpose, we study the interval-valued fuzzy portfolio model based on combination weighting method and score function. The main contributions of this paper are as follows. (1) Combining the interval-valued intuitionistic fuzzy AHP with entropy weight method, we get the combination weighting method. It overcomes the defects of unilateral empowerment law; (2) A new score function is obtained by adjusting the parameters. It can overcome the invalidity of the previous score function for some special interval-valued intuitionistic fuzzy numbers; (3) We propose an interval fuzzy portfolio model based on combination weighting method and new score function, and the theoretical theorem and proof are given. In practical application, a numerical example is given to verify the feasibility and effectiveness of the model.

The rest of this paper is arranged as follows. In Section 2, the basic theory is introduced. In Section 3, the interval-valued intuitionistic fuzzy analytic hierarchy process is introduced. In Section 4, we introduce ten kinds of score functions and accurate functions and their limitations, and construct a new score function. In Section 5, a novel portfolio model with improved interval-valued intuitionistic AHP and score function is constructed, and the feasibility of the model is verified by a numerical example. In Section 6, we summarize the work of this paper.

\section{Some existing definitions and properties}

Definition 1 [12] Suppose int [0,1] is the collection of closed subsets of the interval-valued number $[0,1]$. $X$ is a given theoretical field, $A=\left\{\left\langle x, \mu_{A}(x), v_{A}(x)\right\rangle \mid x \in X\right\}$ is called an interval-valued intuitionistic fuzzy set on the theoretical domain $X . \mu_{A}(x): X \rightarrow \operatorname{int}[0,1]$ and $v_{A}(x): X \rightarrow \operatorname{int}[0,1]$ satisfy $0 \leq \sup \mu_{A}(x)+\sup v_{A}(x) \leq 1, \forall x \in X$. Interval-valued number $\mu_{A}(x)$ is the membership of the element $x . v_{A}(x)$ is the non-membership of the element $x$ :

$$
\left\{\begin{array}{l}
\mu_{A}(x)=\left[\mu_{A}^{L}(x), \mu_{A}^{U}(x)\right], \\
v_{A}(x)=\left[v_{A}^{L}(x), v_{A}^{U}(x)\right] .
\end{array}\right.
$$

The hesitating degree of the element is denoted by

$$
\pi_{A}(x)=\left[\pi_{A}^{L}(x), \pi_{A}^{U}(x)\right]
$$

where

$$
\left\{\begin{array}{l}
\pi_{A}^{L}(x)=1-\mu_{A}^{U}(x)-v_{A}^{U}(x), \\
\pi_{A}^{U}(x)=1-\mu_{A}^{L}(x)-v_{A}^{L}(x) .
\end{array}\right.
$$

Particularly, when $\mu_{A}^{L}(x)=\mu_{A}^{U}(x), \quad v_{A}^{L}(x)=v_{A}^{U}(x)$, the interval-valued intuitionistic fuzzy set degenerates into the intuitionistic fuzzy set. The order interval pairs $\left\langle\mu_{A}(x), v_{A}(x)\right\rangle$ composed of membership interval $\mu_{A}(x)$ and non-membership interval $v_{A}(x)$ is called an interval-valued intuitionistic fuzzy number.

Definition 2 [12] For two interval-valued intuitionistic fuzzy sets

$$
A=\left\{\left\langle x,\left[\mu_{A}^{L}(x), \mu_{A}^{U}(x)\right],\left[v_{A}^{L}(x), v_{A}^{U}(x)\right]\right\rangle \mid x \in X\right\},
$$




$$
B=\left\{\left\langle x,\left[\mu_{B}^{L}(x), \mu_{B}^{U}(x)\right],\left[v_{B}^{L}(x), v_{B}^{U}(x)\right]\right\rangle \mid x \in X\right\},
$$

we have the following relationships

(1) $A \subseteq B \Leftrightarrow \mu_{A}^{L}(x) \leq \mu_{B}^{L}(x), \mu_{A}^{U}(x) \leq \mu_{B}^{U}(x), v_{A}^{L}(x) \geq v_{B}^{L}(x), v_{A}^{U}(x) \geq v_{B}^{U}(x)$;

(2) $A=B \Leftrightarrow A \subseteq B, B \subseteq A$;

(3) $A^{C}=\left\{\left\langle x,\left[v_{A}^{L}(x), v_{A}^{U}(x)\right],\left[\mu_{A}^{L}(x), \mu_{A}^{U}(x)\right]\right\rangle \mid x \in X\right\}$.

Definition 3 [12] For any two interval-valued intuitionistic fuzzy numbers

$$
\alpha_{1}=\left(\left[a_{1}, b_{1}\right],\left[c_{1}, d_{1}\right]\right), \alpha_{2}=\left(\left[a_{2}, b_{2}\right],\left[c_{2}, d_{2}\right]\right),
$$

we have the following operational rules.

(1) $\alpha_{1} \cap \alpha_{2}=\left(\left[\min \left(a_{1}, a_{2}\right), \min \left(b_{1}, b_{2}\right)\right],\left[\max \left(c_{1}, c_{2}\right), \max \left(d_{1}, d_{2}\right)\right]\right)$;

(2) $\alpha_{1} \cup \alpha_{2}=\left(\left[\max \left(a_{1}, a_{2}\right), \max \left(b_{1}, b_{2}\right)\right],\left[\min \left(c_{1}, c_{2}\right), \min \left(d_{1}, d_{2}\right)\right]\right)$;

(3) $\alpha_{1}+\alpha_{2}=\left(\left[a_{1}+a_{2}-a_{1} a_{2}, b_{1}+b_{2}-b_{1} b_{2}\right],\left[c_{1} c_{2}, d_{1} d_{2}\right]\right)$;

(4) $\alpha_{1} \cdot \alpha_{2}=\left(\left[a_{1} a_{2}, b_{1} b_{2}\right],\left[c_{1}+c_{2}-c_{1} c_{2}, d_{1}+d_{2}-d_{1} d_{2}\right]\right)$;

(5) $\lambda \alpha_{1}=\left(\left[1-\left(1-a_{1}\right)^{\lambda}, 1-\left(1-b_{1}\right)^{\lambda}\right],\left[c_{1}^{\lambda}, d_{1}^{\lambda}\right]\right), \lambda>0$;

(6) $\alpha_{1}^{\lambda}=\left(\left[a_{1}^{\lambda}, b_{1}^{\lambda}\right],\left[1-\left(1-c_{1}\right)^{\lambda}, 1-\left(1-d_{1}\right)^{\lambda}\right]\right), \lambda>0$.

Definition 4 [12] Suppose $\alpha_{j}=\left(\left[a_{j}, b_{j}\right],\left[c_{j}, d_{j}\right]\right)(j=1,2, \ldots, n)$ is a set of interval-valued intuitionistic fuzzy numbers, then

$$
\begin{aligned}
\operatorname{IIFWA_{\omega }}\left(\alpha_{1}, \alpha_{2}, \ldots, \alpha_{n}\right) & =\sum_{j=1}^{n} \omega_{j} \alpha_{j} \\
& =\left(\left[1-\prod_{j=1}^{n}\left(1-a_{j}\right)^{\omega_{j}}, 1-\prod_{j=1}^{n}\left(1-b_{j}\right)^{\omega_{j}}\right],\left[\prod_{j=1}^{n} c_{j}^{\omega_{j}}, \prod_{j=1}^{n} d_{j}^{\omega_{j}}\right]\right),
\end{aligned}
$$

which is called interval-valued intuitionistic fuzzy weighted arithmetic average operator;

$$
\begin{aligned}
\operatorname{IIFWG}\left(\alpha_{\omega}, \alpha_{2}, \ldots, \alpha_{n}\right) & =\sum_{j=1}^{n} \omega_{j} \alpha_{j} \\
& =\left(\left[\prod_{j=1}^{n} a_{j}^{\omega_{j}}, \prod_{j=1}^{n} b_{j}^{\omega_{j}}\right],\left[1-\prod_{j=1}^{n}\left(1-c_{j}\right)^{\omega_{j}}, 1-\prod_{j=1}^{n}\left(1-d_{j}\right)^{\omega_{j}}\right]\right),
\end{aligned}
$$

which is called interval-valued intuitionistic fuzzy weighted geometric average operator. $\omega_{j}(j=1,2, \ldots, n)$ is the weight vector of $\alpha_{j}(j=1,2, \ldots, n)$, where $0<\omega_{j}<1$ and $\sum_{j=1}^{n} \omega_{j}=1$. Specially, when $\omega_{j}=\frac{1}{n}(j=1,2, \ldots, n), \quad I I F W A_{\omega}\left(\alpha_{1}, \alpha_{2}, \ldots, \alpha_{n}\right)$ and $I I F W G_{\omega}\left(\alpha_{1}, \alpha_{2}, \ldots, \alpha_{n}\right)$ degenerate into interval-valued intuitionistic fuzzy arithmetic average operator $\operatorname{IIFA}_{\omega}\left(\alpha_{1}, \alpha_{2}, \ldots, \alpha_{n}\right) \quad$ and interval-valued intuitionistic fuzzy geometric average operator $I I F G_{\omega}\left(\alpha_{1}, \alpha_{2}, \ldots, \alpha_{n}\right)$ respectively. That is 


$$
\begin{aligned}
\operatorname{IIFA_{\omega }}\left(\alpha_{1}, \alpha_{2}, \ldots, \alpha_{n}\right) & =\sum_{j=1}^{n} \omega_{j} \alpha_{j} \\
& =\left(\left[1-\prod_{j=1}^{n}\left(1-a_{j}\right)^{\frac{1}{n}}, 1-\prod_{j=1}^{n}\left(1-b_{j}\right)^{\frac{1}{n}}\right],\left[\prod_{j=1}^{n} c_{j}^{\frac{1}{n}}, \prod_{j=1}^{n} d_{j}^{\frac{1}{n}}\right]\right), \\
I I F G_{\omega}\left(\alpha_{1}, \alpha_{2}, \ldots, \alpha_{n}\right) & =\sum_{j=1}^{n} \omega_{j} \alpha_{j} \\
& =\left(\left[\prod_{j=1}^{n} a_{j}^{\frac{1}{n}}, \prod_{j=1}^{n} b_{j}^{\frac{1}{n}}\right],\left[1-\prod_{j=1}^{n}\left(1-c_{j}\right)^{\frac{1}{n}}, 1-\prod_{j=1}^{n}\left(1-d_{j}\right)^{\frac{1}{n}}\right]\right) .
\end{aligned}
$$

\section{Some new definition, theorems and relative proofs}

\subsection{A new theorem for interval-valued intuitionistic fuzzy number}

Theorem 1 For any three interval-valued intuitionistic fuzzy numbers

$$
\alpha_{1}=\left(\left[a_{1}, b_{1}\right],\left[c_{1}, d_{1}\right]\right), \alpha_{2}=\left(\left[a_{2}, b_{2}\right],\left[c_{2}, d_{2}\right]\right), \alpha_{3}=\left(\left[a_{3}, b_{3}\right],\left[c_{3}, d_{3}\right]\right),
$$

we have the following properties.

(1) Closure property: $\alpha_{1}+\alpha_{2}$ and $\alpha_{1} \alpha_{2}$ are both interval-valued intuitionistic fuzzy numbers;

(2) Commutative law: $\alpha_{1}+\alpha_{2}=\alpha_{2}+\alpha_{1}, \alpha_{1} \cdot \alpha_{2}=\alpha_{2} \cdot \alpha_{1}$;

(3) Associative law: $\alpha_{1}+\alpha_{2}+\alpha_{3}=\alpha_{1}+\left(\alpha_{2}+\alpha_{3}\right), \alpha_{1} \alpha_{2} \alpha_{3}=\alpha_{1}\left(\alpha_{2} \alpha_{3}\right)$;

(4) De Morgan law: $\left(\alpha_{1}+\alpha_{2}\right)^{C}=\alpha_{1}^{C} \alpha_{2}^{C},\left(\alpha_{1} \alpha_{2}\right)^{C}=\alpha_{1}^{C}+\alpha_{2}^{C}$.

Our above proof process is inspired by Atanassov K.T. and Gargov G [12].

\section{Proof:}

(1) If we have $\mu_{\alpha_{1}+\alpha_{2}}(x) \subseteq[0,1], v_{\alpha_{1}+\alpha_{2}}(x) \subseteq[0,1], \sup \mu_{\alpha_{1}+\alpha_{2}}(x)+\sup v_{\alpha_{1}+\alpha_{2}}(x) \leq 1$, then we can prove that $\alpha_{1}+\alpha_{2}$ is the interval-valued intuitionistic fuzzy number.

Since

$$
\begin{aligned}
\alpha_{1}+\alpha_{2}= & \left(\left[a_{1}+a_{2}-a_{1} a_{2}, b_{1}+b_{2}-b_{1} b_{2}\right],\left[c_{1} c_{2}, d_{1} d_{2}\right]\right) \\
= & \left(\left[1-\left(1-a_{1}\right)\left(1-a_{2}\right), 1-\left(1-b_{1}\right)\left(1-b_{2}\right)\right],\left[c_{1} c_{2}, d_{1} d_{2}\right]\right), \\
& 0 \leq a_{1} \leq b_{1} \leq 1,0 \leq a_{2} \leq b_{2} \leq 1,
\end{aligned}
$$

we have

$$
0 \leq 1-\left(1-a_{1}\right)\left(1-a_{2}\right) \leq 1, \quad 0 \leq 1-\left(1-b_{1}\right)\left(1-b_{2}\right) \leq 1 .
$$

By (5), we have

$$
1-\left(1-a_{1}\right)\left(1-a_{2}\right) \leq 1-\left(1-b_{1}\right)\left(1-b_{2}\right) .
$$

Correspondingly, we obtain

$$
a_{1}+a_{2}-a_{1} a_{2} \leq b_{1}+b_{2}-b_{1} b_{2} .
$$

By given conditions, it is obvious that

$$
0 \leq c_{1} \leq d_{1} \leq 1,0 \leq c_{2} \leq d_{2} \leq 1,
$$

then

$$
0 \leq c_{1} c_{2} \leq 1,0 \leq d_{1} d_{2} \leq 1 .
$$


Since

$$
\begin{gathered}
c_{1} c_{2} \leq d_{1} d_{2}, \\
b_{1}+b_{2}-b_{1} b_{2}+d_{1} d_{2}=1-\left(1-b_{1}\right)\left(1-b_{2}\right)+d_{1} d_{2}, \\
b_{1}+d_{1} \leq 1, b_{2}+d_{2} \leq 1,
\end{gathered}
$$

we can obtain

$$
1-b_{1} \geq d_{1}, 1-b_{2} \geq d_{2} \text {. }
$$

Then

$$
\begin{gathered}
\left(1-b_{1}\right)\left(1-b_{2}\right) \geq d_{1} d_{2}, \\
1-\left(1-b_{1}\right)\left(1-b_{2}\right)+d_{1} d_{2} \leq 1 .
\end{gathered}
$$

We can obtain that $\alpha_{1}+\alpha_{2}$ is the interval-valued intuitionistic fuzzy number.

Similarly, we also can prove that $\alpha_{1} \alpha_{2}$ is the interval-valued intuitionistic fuzzy number.

(2) Since

$$
\begin{aligned}
& \alpha_{1}+\alpha_{2}=\left(\left[a_{1}+a_{2}-a_{1} a_{2}, b_{1}+b_{2}-b_{1} b_{2}\right],\left[c_{1} c_{2}, d_{1} d_{2}\right]\right), \\
& \alpha_{2}+\alpha_{1}=\left(\left[a_{2}+a_{1}-a_{2} a_{1}, b_{2}+b_{1}-b_{2} b_{1}\right],\left[c_{2} c_{1}, d_{2} d_{1}\right]\right),
\end{aligned}
$$

we have $\alpha_{1}+\alpha_{2}=\alpha_{2}+\alpha_{1}$.

Since

$$
\begin{aligned}
& \alpha_{1} \cdot \alpha_{2}=\left(\left[a_{1} a_{2}, b_{1} b_{2}\right],\left[c_{1}+c_{2}-c_{1} c_{2}, d_{1}+d_{2}-d_{1} d_{2}\right]\right), \\
& \alpha_{2} \cdot \alpha_{1}=\left(\left[a_{2} a_{1}, b_{2} b_{1}\right],\left[c_{2}+c_{1}-c_{2} c_{1}, d_{2}+d_{1}-d_{2} d_{1}\right]\right),
\end{aligned}
$$

we have $\alpha_{1} \cdot \alpha_{2}=\alpha_{2} \cdot \alpha_{1}$.

(3) On the one hand, since

$$
\begin{gathered}
\alpha_{1}+\alpha_{2}=\left(\left[a_{1}+a_{2}-a_{1} a_{2}, b_{1}+b_{2}-b_{1} b_{2}\right],\left[c_{1} c_{2}, d_{1} d_{2}\right]\right), \\
\alpha_{2}+\alpha_{3}=\left(\left[a_{2}+a_{3}-a_{2} a_{3}, b_{2}+b_{3}-b_{2} b_{3}\right],\left[c_{2} c_{3}, d_{2} d_{3}\right]\right),
\end{gathered}
$$

we have

$$
\begin{aligned}
& \alpha_{1}+\alpha_{2}+\alpha_{3} \\
= & \left.\left(\left[a_{1}+a_{2}-a_{1} a_{2}+a_{3}-\left(a_{1}+a_{2}-a_{1} a_{2}\right) a_{3}, b_{1}+b_{2}-b_{1} b_{2}+b_{3}-\left(b_{1}+b_{2}-b_{1} b_{2}\right) b_{3}\right],\left[c_{1} c_{2} c_{3}, d_{1} d_{2} d_{3}\right]\right)\right) \\
= & \left(\left[a_{1}+a_{2}+a_{3}-a_{1} a_{2}-a_{1} a_{3}-a_{2} a_{3}+a_{1} a_{2} a_{3}, b_{1}+b_{2}+b_{3}-b_{1} b_{2}-b_{1} b_{3}-b_{2} b_{3}+b_{1} b_{2} b_{3}\right],\left[c_{1} c_{2} c_{3}, d_{1} d_{2} d_{3}\right]\right) .
\end{aligned}
$$

On the other hand, we obtain

$$
\begin{aligned}
& \alpha_{1}+\left(\alpha_{2}+\alpha_{3}\right) \\
= & \left(\left[a_{1}+a_{2}+a_{3}-a_{2} a_{3}-a_{1}\left(a_{2}+a_{3}-a_{2} a_{3}\right), b_{1}+b_{2}+b_{3}-b_{2} b_{3}-b_{1}\left(b_{2}+b_{3}-b_{2} b_{3}\right)\right],\left[c_{1} c_{2} c_{3}, d_{1} d_{2} d_{3}\right]\right) \\
= & \left.\left.\left(\left[a_{1}+a_{2}+a_{3}-a_{2} a_{3}-a_{1} a_{2}-a_{1} a_{3}+a_{1} a_{2} a_{3}\right), b_{1}+b_{2}+b_{3}-b_{2} b_{3}-b_{1} b_{2}-b_{1} b_{3}+b_{1} b_{2} b_{3}\right)\right],\left[c_{1} c_{2} c_{3}, d_{1} d_{2} d_{3}\right]\right) \\
= & \left.\left.\left(\left[a_{1}+a_{2}+a_{3}-a_{2} a_{3}-a_{1} a_{2}-a_{1} a_{3}+a_{1} a_{2} a_{3}\right), b_{1}+b_{2}+b_{3}-b_{2} b_{3}-b_{1} b_{2}-b_{1} b_{3}+b_{1} b_{2} b_{3}\right)\right],\left[c_{1} c_{2} c_{3}, d_{1} d_{2} d_{3}\right]\right),
\end{aligned}
$$

so, we have

$$
\alpha_{1}+\alpha_{2}+\alpha_{3}=\alpha_{1}+\left(\alpha_{2}+\alpha_{3}\right) .
$$

On the one hand, since

$$
\alpha_{1} \cdot \alpha_{2}=\left(\left[a_{1} a_{2}, b_{1} b_{2}\right],\left[c_{1}+c_{2}-c_{1} c_{2}, d_{1}+d_{2}-d_{1} d_{2}\right]\right),
$$




$$
\alpha_{2} \cdot \alpha_{3}=\left(\left[a_{2} a_{3}, b_{2} b_{3}\right],\left[c_{2}+c_{3}-c_{2} c_{3}, d_{2}+d_{3}-d_{2} d_{3}\right]\right),
$$

we have

$$
\begin{aligned}
& \alpha_{1} \alpha_{2} \alpha_{3} \\
= & \left(\left[a_{1} a_{2} a_{3}, b_{1} b_{2} b_{3}\right],\left[c_{1}+c_{2}-c_{1} c_{2}+c_{3}-\left(c_{1}+c_{2}-c_{1} c_{2}\right) c_{3}, d_{1}+d_{2}-d_{1} d_{2}+d_{3}-\left(d_{1}+d_{2}-d_{1} d_{2}\right) d_{3}\right]\right) \\
= & \left(\left[a_{1} a_{2} a_{3}, b_{1} b_{2} b_{3}\right],\left[c_{1}+c_{2}+c_{3}-c_{1} c_{2}-c_{1} c_{3}-c_{2} c_{3}+c_{1} c_{2} c_{3}, d_{1}+d_{2}+d_{3}-d_{1} d_{2}-d_{1} d_{3}-d_{2} d_{3}+d_{1} d_{2} d_{3}\right]\right) .
\end{aligned}
$$

On the other hand, we obtain

$$
\begin{aligned}
& \alpha_{1}\left(\alpha_{2} \alpha_{3}\right) \\
= & \left(\left[a_{1} a_{2} a_{3}, b_{1} b_{2} b_{3}\right],\left[c_{1}+c_{2}+c_{3}-c_{2} c_{3}-c_{1}\left(c_{2}+c_{3}-c_{2} c_{3}\right), d_{1}+d_{2}+d_{3}-d_{2} d_{3}-d_{1}\left(d_{2}+d_{3}-d_{2} d_{3}\right)\right]\right) \\
= & \left(\left[a_{1} a_{2} a_{3}, b_{1} b_{2} b_{3}\right],\left[c_{1}+c_{2}+c_{3}-c_{2} c_{3}-c_{1} c_{2}-c_{1} c_{3}+c_{1} c_{2} c_{3}, d_{1}+d_{2}+d_{3}-d_{2} d_{3}-d_{1} d_{2}-d_{1} d_{3}+d_{1} d_{2} d_{3}\right]\right),
\end{aligned}
$$

we have

$$
\alpha_{1} \alpha_{2} \alpha_{3}=\alpha_{1}\left(\alpha_{2} \alpha_{3}\right)
$$

(4) Since

$$
\begin{gathered}
\left(\alpha_{1}+\alpha_{2}\right)^{C}=\left(\left[a_{1}+a_{2}-a_{1} a_{2}, b_{1}+b_{2}-b_{1} b_{2}\right],\left[c_{1} c_{2}, d_{1} d_{2}\right]\right)^{C} \\
=\left(\left[c_{1} c_{2}, d_{1} d_{2}\right],\left[a_{1}+a_{2}-a_{1} a_{2}, b_{1}+b_{2}-b_{1} b_{2}\right]\right), \\
\alpha_{1}^{C}=\left(\left[c_{1}, d_{1}\right],\left[a_{1}, b_{1}\right]\right), \\
\alpha_{2}^{C}=\left(\left[c_{2}, d_{2}\right],\left[a_{2}, b_{2}\right]\right),
\end{gathered}
$$

we can obtain

$$
\left(\alpha_{1}+\alpha_{2}\right)^{C}=\alpha_{1}^{C} \alpha_{2}^{C}
$$

Similarly, we also can prove

$$
\left(\alpha_{1} \alpha_{2}\right)^{C}=\alpha_{1}^{C}+\alpha_{2}^{C} \text {. }
$$

Remark 1 It should be noted that the following equation does not hold:

$$
\alpha_{1}\left(\alpha_{2}+\alpha_{3}\right)=\alpha_{1} \alpha_{2}+\alpha_{1} \alpha_{3} .
$$

Let

we have

$$
\alpha_{1}=([0.1,0.2],[0.5,0.7]), \alpha_{2}=([0.2,0.3],[0.4,0.6]), \alpha_{3}=([0.1,0.3],[0.1,0.2]),
$$

$$
\alpha_{1}\left(\alpha_{2}+\alpha_{3}\right)=([0.028,0.102],[0.04,0.12]), \alpha_{1} \alpha_{2}+\alpha_{1} \alpha_{3}=([0.098,0.1164],[0.385,0.6688]) .
$$

Obviously, we can see that

$$
\alpha_{1}\left(\alpha_{2}+\alpha_{3}\right) \neq \alpha_{1} \alpha_{2}+\alpha_{1} \alpha_{3}
$$

\subsection{A new division definition and related proof}

Definition 5 For any two interval-valued intuitionistic fuzzy numbers $\alpha_{1}=\left(\left[a_{1}, b_{1}\right],\left[c_{1}, d_{1}\right]\right)$ and $\alpha_{2}=\left(\left[a_{2}, b_{2}\right],\left[c_{2}, d_{2}\right]\right)$, we can define the subtraction operator and division operator:

$$
\alpha_{1}-\alpha_{2}=\alpha_{1} \alpha_{2}^{C}=\left(\left[a_{1} c_{2}, b_{1} d_{2}\right],\left[c_{1}+a_{2}-c_{1} a_{2}, d_{1}+b_{2}-d_{1} b_{2}\right]\right),
$$




$$
\alpha_{1} \div \alpha_{2}=\alpha_{1}+\alpha_{2}^{C}=\left(\left[a_{1}+c_{2}-a_{1} c_{2}, b_{1}+d_{2}-b_{1} d_{2}\right],\left[c_{1} a_{2}, d_{1} b_{2}\right]\right) .
$$

Since subtraction comes from multiplication and division from addition, $\alpha_{1}-\alpha_{2}$ and $\alpha_{1} \div \alpha_{2}$ are both interval-valued intuitionistic fuzzy numbers.

Remark 2 Referring to the definition of division of intuitionistic fuzzy numbers, we give the definition of division of interval intuitionistic fuzzy numbers. Then we prove its rationality. This can simplify the division operation of interval-valued intuitionistic fuzzy numbers.

Theorem 2 For the interval-valued intuitionistic fuzzy number

$$
\alpha_{1}=\left(\left[a_{1}, b_{1}\right],\left[c_{1}, d_{1}\right]\right), \alpha_{2}=\left(\left[a_{2}, b_{2}\right],\left[c_{2}, d_{2}\right]\right), \alpha_{3}=\left(\left[a_{3}, b_{3}\right],\left[c_{3}, d_{3}\right]\right),
$$

we have the following two properties.

(1) $\left(\alpha_{1}-\alpha_{2}\right)-\alpha_{3}=\alpha_{1}-\left(\alpha_{2}+\alpha_{3}\right)$;

(2) $\left(\alpha_{1} \div \alpha_{2}\right) \div \alpha_{3}=\alpha_{1} \div\left(\alpha_{2} \alpha_{3}\right)$.

Proof:

(1) According to Definition 5 and Theorem 1, we can get

$$
\left(\alpha_{1}-\alpha_{2}\right)-\alpha_{3}=\left(\alpha_{1} \alpha_{2}^{C}\right) \alpha_{3}^{C}=\alpha_{1}\left(\alpha_{2}^{C} \alpha_{3}^{C}\right)=\alpha_{1}\left(\alpha_{2}+\alpha_{3}\right)^{C}=\alpha_{1}-\left(\alpha_{2}+\alpha_{3}\right) .
$$

(2) According to Definition 5 and Theorem 1, we can also get

$$
\left(\alpha_{1} \div \alpha_{2}\right) \div \alpha_{3}=\left(\alpha_{1}+\alpha_{2}^{C}\right)+\alpha_{3}^{C}=\alpha_{1}+\left(\alpha_{2}^{C}+\alpha_{3}^{C}\right)=\alpha_{1}+\left(\alpha_{2} \alpha_{3}\right)^{C}=\alpha_{1} \div\left(\alpha_{2} \alpha_{3}\right) \text {. }
$$

\section{The ideas and steps of our new interval-valued intuitionistic fuzzy AHP}

With the help of the idea of intuitionistic fuzzy AHP of Xu [21], we can construct interval-valued intuitionistic AHP, the basic ideas and specific steps are as follows.

\subsection{The basic ideas}

Our inspiration comes from Xu [21]'s research on intuitive AHP. He extended the classical AHP and the fuzzy AHP to the context of IFS, where the scale of pairwise comparisons of the decision maker was represented by intuitionistic fuzzy numbers. He established a perfect multiplicative consistent interval-valued intuitionistic fuzzy judgment matrix to check whether the preference relation is consistent or not. The weight vector of the intuitionistic preference relation could be derived through a normalizing rank summation method. Based on the weight vector and score function, the score of each index was obtained and finally obtained the normalized weight vector. Then we try to extend the AHP to the context of interval-valued intuitionistic fuzzy set, which is powerful in describing vagueness and uncertainty.

\subsection{The specific steps}

Step 1: Establish an interval-valued intuitionistic fuzzy judgment matrix.

First, we establish an interval-valued intuitionistic fuzzy judgment matrix $R=\left(r_{i j}\right)_{n \times m}$, where $r_{i j}=\left(\left[a_{i j}, b_{i j}\right],\left[c_{i j}, d_{i j}\right]\right),\left[a_{i j}, b_{i j}\right]=\left[c_{j i}, d_{j i}\right],\left[c_{i j}, d_{i j}\right]=\left[a_{j i}, b_{j i}\right]$ and $\left[a_{i i}, b_{i i}\right]=\left[c_{i i}, d_{i i}\right]=[0.5,0.5] . \quad\left[a_{i j}, b_{i j}\right]$ indicates the extent to which decision makers prefer the $i$-th index to the $j$-th index, $\left[c_{i j}, d_{i j}\right]$ indicates the extent to which decision makers prefer the $j$-th index to the $i$-th index. Preference information can be compared between two indexes using the scaling method [22]. 
Table 1 Scaling meaning

\begin{tabular}{ccc}
\hline Criterion & $\mu$ & $v$ \\
\hline absolutely low & {$[0.10,0.25]$} & {$[0.65,0.75]$} \\
very low & {$[0.15,0.30]$} & {$[0.60,0.70]$} \\
low & {$[0.20,0.35]$} & {$[0.55,0.65]$} \\
on the low side & {$[0.25,0.40]$} & {$[0.50,0.60]$} \\
approximately equal & {$[0.45,0.55]$} & {$[0.30,0.45]$} \\
absolutely equal & {$[0.50,0.50]$} & {$[0.50,0.50]$} \\
on the high side & {$[0.50,0.60]$} & {$[0.25,0.40]$} \\
high & {$[0.55,0.65]$} & {$[0.20,0.35]$} \\
very high & {$[0.60,0.70]$} & {$[0.15,0.30]$} \\
absolute height & {$[0.65,0.75]$} & {$[0.10,0.25]$} \\
\hline
\end{tabular}

Step 2: Check the consistency by establishing consistent matrix $\bar{R}$.

We can check the consistency of $R=\left(r_{i j}\right)_{n \times m}$ by establishing a perfect multiplicative consistent interval-valued intuitionistic fuzzy judgment matrix $\bar{R}=\left(\overline{r_{i j}}\right)_{n \times m}$. There are three possible cases:

Case 1: When $j>i+1$, let $\overline{r_{i j}}=\left(\left[\overline{a_{i j}} \overline{b_{i j}}\right],\left[\overline{c_{i j}}, \overline{d_{i j}}\right]\right)$;

Case 2: When $j=i+1$ or $j=i$, let $\overline{r_{i j}}=r_{i j}$;

Case 3: When $j<i$, let $\overline{r_{i j}}=\left(\left[\overline{c_{i j}}, \overline{d_{i j}}\right],\left[\overline{a_{i j}}, \overline{b_{i j}}\right]\right)$.

Where

$$
\begin{aligned}
& \int \overline{a_{i j}}=\frac{\sqrt[j-i-1]{\prod_{t=i+1}^{j-1} a_{i t} a_{t j}}}{\sqrt[j-i-1]{\prod_{t=i+1}^{j-1} a_{i t} a_{t j}}+\sqrt[j-i-1]{\prod_{t=i+1}^{j-1}\left(1-a_{i t}\right)\left(1-a_{t j}\right)}}, \\
& \overline{b_{i j}}=\frac{\sqrt[j-i-1]{\prod_{t=i+1}^{j-1} b_{i t} b_{t j}}}{\sqrt[j-i-1]{\prod_{t=i+1}^{j-1} b_{i t} b_{t j}}+\sqrt[j-i-1]{\prod_{t=i+1}^{j-1}\left(1-b_{i t}\right)\left(1-b_{t j}\right)}}, \\
& \overline{c_{i j}}=\frac{\sqrt[j-i-1]{\prod_{t=i+1}^{j-1} c_{i t} c_{t j}}}{\sqrt[j-i-1]{\prod_{t=i+1}^{j-1} c_{i t} c_{t j}}+\sqrt[j-i-1]{\prod_{t=i+1}^{j-1}\left(1-c_{i t}\right)\left(1-c_{t j}\right)}}, \\
& \overline{d_{i j}}=\frac{\sqrt[j-i-1]{\prod_{t=i+1}^{j-1} d_{i t} d_{t j}}}{\sqrt[j-i-11]{\prod_{t=i+1}^{j-1} d_{i t} d_{t j}}+\sqrt[j-i-1]{\prod_{t=i+1}^{j-1}\left(1-d_{i t}\right)\left(1-d_{t j}\right)}} .
\end{aligned}
$$

The consistency of $R$ is acceptable if $R$ and $\bar{R}$ meet the following conditions: $d(R, \bar{R})<\tau$, where $\tau$ is a consistency threshold. Generally, its value is $0.1 . d(R, \bar{R})$ is the distance measure for $R$ to $\bar{R}$, where

$$
d(R, \bar{R})=\frac{1}{4(n-1)(n-2)} \sum_{i=1}^{n} \sum_{j=1}^{n} \begin{aligned}
& \left(\left|\overline{a_{i j}}-a_{i j}\right|+\left|\overline{b_{i j}}-b_{i j}\right|+\left|\overline{c_{i j}}-c_{i j}\right|+\left|\overline{d_{i j}}-d_{i j}\right|\right. \\
& \left.+\left|\overline{1-a_{i j}-c_{i j}}-\left(1-a_{i j}-c_{i j}\right)\right|+\left|\overline{1-b_{i j}-d_{i j}}-\left(1-b_{i j}-d_{i j i j}\right)\right|\right) .
\end{aligned}
$$

Step 3: Calculate the weights of the indexes by introducing parameters.

For the interval-valued intuitionistic fuzzy number $r_{i j}=\left(\left[a_{i j}, b_{i j}\right],\left[c_{i j}, d_{i j}\right]\right)$, let $r_{i j}{ }^{\prime}=\left(m_{i j}, n_{i j}\right)$, preference parameters 


$$
\begin{gathered}
m_{i j}\left(\lambda_{i j}\right)=a_{i j}+\lambda_{i j}\left(b_{i j}-a_{i j}\right), \\
n_{i j}\left(\lambda_{i j}\right)=c_{i j}+\left(1-\lambda_{i j}\right)\left(d_{i j}-c_{i j}\right),
\end{gathered}
$$

where $m_{i j}$ represents the parameter membership of the interval-valued intuitionistic fuzzy number $r_{i j}{ }^{\prime}$, $n_{i j}$ represents the parameter non-membership of the interval-valued intuitionistic fuzzy number $r_{i j}{ }^{\prime}$. $\lambda_{i j}$ represents the satisfaction coefficient of decision maker to the $i$-th index relative to the $j$-th index and $\lambda_{i j} \in[0,1]$. The larger the value of $\lambda_{i j}$ is, the more satisfied the decision maker is with the $i$-th index. Then $r_{i j}=\left(\left[a_{i j}, b_{i j}\right],\left[c_{i j}, d_{i j}\right]\right)$ can be converted to

$$
r_{i j}{ }^{\prime}=\left(m_{i j}, n_{i j}\right)=\left(a_{i j}+\lambda_{i j}\left(b_{i j}-a_{i j}\right), c_{i j}+\left(1-\lambda_{i j}\right)\left(d_{i j}-c_{i j}\right)\right) .
$$

Since $m_{i j}+n_{i j}=a_{i j}+\lambda_{i j}\left(b_{i j}-a_{i j}\right)+c_{i j}+\left(1-\lambda_{i j}\right)\left(d_{i j}-c_{i j}\right) \leq b_{i j}+d_{i j} \leq 1$, thus $m_{i j} \leq 1-n_{i j}$, we can convert $\left(m_{i j}, n_{i j}\right)$ to $\left[m_{i j}, 1-n_{i j}\right]$, then we can convert $R^{\prime}=\left(r_{i j}^{\prime}\right)_{n \times m}=\left(m_{i j}, n_{i j}\right)_{n \times m}$ to $R^{\prime \prime}=\left(r_{i j}^{\prime \prime}\right)_{n \times m}=\left(\left[m_{i j}, 1-n_{i j}\right]\right)_{n \times m}$. According to the arithmetic rules of the interval, we use normalizing rank summation method [21] to obtain the weights as follows:

$$
\omega^{\prime}=\frac{\sum_{j=1}^{n} r_{i j}^{\prime \prime}}{\sum_{i=1}^{n} \sum_{j=1}^{n} r_{i j}^{\prime \prime}}=\frac{\sum_{j=1}^{n}\left[m_{i j}, 1-n_{i j}\right]}{\sum_{i=1}^{n} \sum_{j=1}^{n}\left[m_{i j}, 1-n_{i j}\right]}=\frac{\left[\sum_{j=1}^{n} m_{i j}, \sum_{j=1}^{n}\left(1-n_{i j}\right)\right]}{\left[\sum_{i=1}^{n} \sum_{j=1}^{n} m_{i j}, \sum_{i=1}^{n} \sum_{j=1}^{n}\left(1-n_{i j}\right)\right]}=\left[\frac{\sum_{j=1}^{n} m_{i j}}{\sum_{i=1}^{n} \sum_{j=1}^{n}\left(1-n_{i j}\right)}, \frac{\sum_{j=1}^{n}\left(1-n_{i j}\right)}{\sum_{i=1}^{n} \sum_{j=1}^{n} m_{i j}}\right] .
$$

Then we convert the interval $\left[\frac{\sum_{j=1}^{n} m_{i j}}{\sum_{i=1}^{n} \sum_{j=1}^{n}\left(1-n_{i j}\right)}, \frac{\sum_{j=1}^{n}\left(1-n_{i j}\right)}{\sum_{i=1}^{n} \sum_{j=1}^{n} m_{i j}}\right]$ to its corresponding intuitionistic fuzzy number. Finally, we have

$$
\omega_{i}^{\prime}=\left(\frac{\sum_{j=1}^{n} m_{i j}}{\sum_{i=1}^{n} \sum_{j=1}^{n}\left(1-n_{i j}\right)}, 1-\frac{\sum_{j=1}^{n}\left(1-n_{i j}\right)}{\sum_{i=1}^{n} \sum_{j=1}^{n} m_{i j}}\right)
$$

Next, we can show that $0 \leq \frac{\sum_{j=1}^{n} m_{i j}}{\sum_{i=1}^{n} \sum_{j=1}^{n}\left(1-n_{i j}\right)} \leq 1,0 \leq 1-\frac{\sum_{j=1}^{n}\left(1-n_{i j}\right)}{\sum_{i=1}^{n} \sum_{j=1}^{n} m_{i j}} \leq 1$.

In fact, $\quad$ since $\quad \sum_{j=1}^{n} a_{i j} \leq \sum_{j=1}^{n} m_{i j} \leq \sum_{j=1}^{n} b_{i j} \quad, \quad \sum_{j=1}^{n} c_{i j} \leq \sum_{j=1}^{n} n_{i j} \leq \sum_{j=1}^{n} d_{i j} \quad$ and $\quad b_{i j} \leq 1-d_{i j} \quad$, then $\quad$ we have $\sum_{j=1}^{n} a_{i j} \leq \sum_{j=1}^{n} m_{i j} \leq \sum_{j=1}^{n} b_{i j} \leq \sum_{j=1}^{n}\left(1-d_{i j}\right) \leq \sum_{j=1}^{n}\left(1-n_{i j}\right) \leq \sum_{j=1}^{n}\left(1-c_{i j}\right)$. So $0 \leq \frac{\sum_{j=1}^{n} m_{i j}}{\sum_{i=1}^{n} \sum_{j=1}^{n}\left(1-n_{i j}\right)} \leq 1$. Similarly, we can show that $0 \leq 1-\frac{\sum_{j=1}^{n}\left(1-n_{i j}\right)}{\sum_{i=1}^{n} \sum_{j=1}^{n} m_{i j}} \leq 1$.

Step 4: Sort the indexes. 
We sort the indicators using the score function. Set the score function as follows.

$$
f(\alpha)=\frac{1-v_{\alpha}}{1+\pi_{\alpha}} .
$$

Step 5: Normalize the weights.

We calculate the normalized weights of each indicator according to Formula (56).

$$
\omega_{i}=\frac{f\left(\omega_{i}^{\prime}\right)}{\sum_{i=1}^{n} f\left(\omega_{i}^{\prime}\right)} .
$$

Remark 3: Intuitionistic fuzzy AHP proposed by Xu [21] applied AHP to intuitionistic fuzzy sets. We extend it to interval-valued intuitionistic fuzzy sets and obtain the internal intuitionistic AHP. When establishing the interval-valued intuitionistic fuzzy judgment matrix, we change the intuitionistic fuzzy assessments $r_{i j}=\left(\mu_{i j}, v_{i j}\right)$ into interval-valued intuitionistic fuzzy assessments $r_{i j}=\left(\left[a_{i j}, b_{i j}\right],\left[c_{i j}, d_{i j}\right]\right)$. When doing the consistency check, we also propose new $\bar{R}=\left(\overline{r_{i j}}\right)_{n \times m}$ and $d(R, \bar{R})$.

Remark 4: When we calculate the weight of the evaluation index, we introduce a parameter $\lambda_{i j}$ to express the satisfaction coefficient of decision maker to the $i$-th index compared with the $j$-th index, and then we can change an interval-valued intuitionistic fuzzy number into an intuitionistic fuzzy number.

Schematic diagram of the IVIFAHP is as follows:

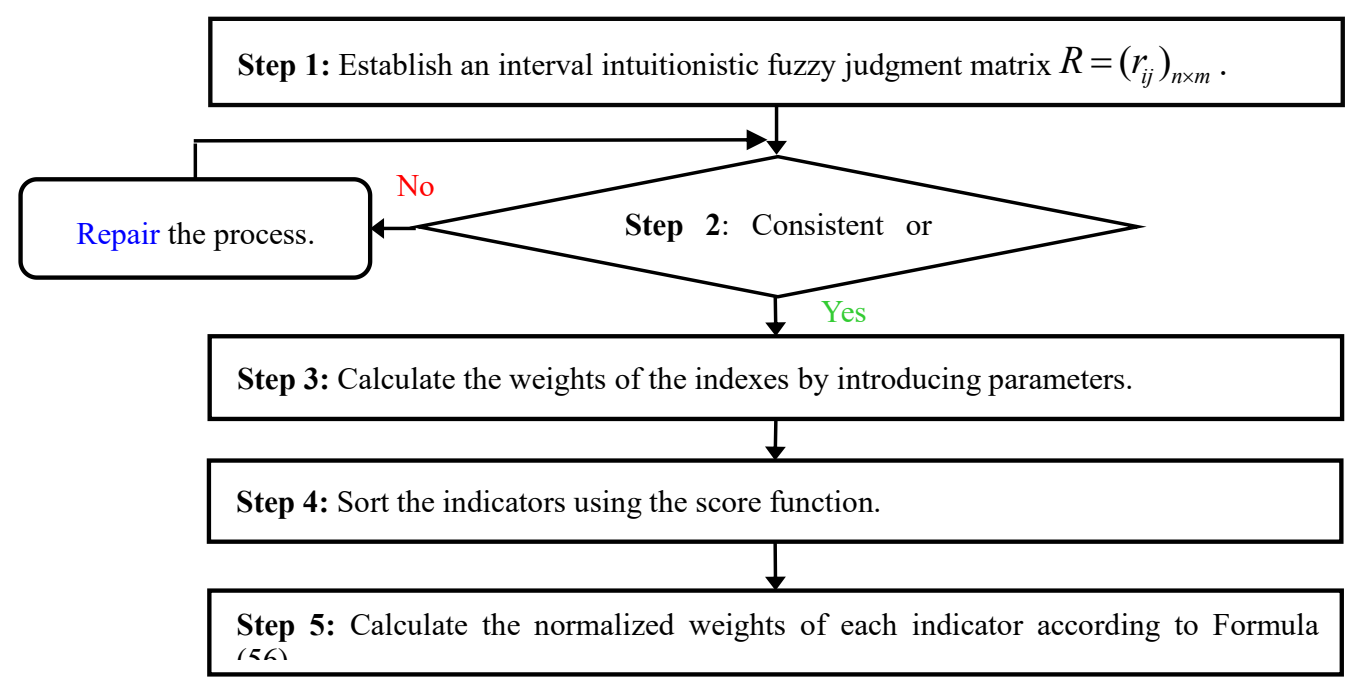

Figure 1: Schematic diagram of the IVIFAHP.

\section{The limitation and improvement analysis of score functions}

In the previous section, we have already introduced the construction of interval-valued intuitionistic fuzzy hierarchical analysis. In this chapter, we will introduce the existing score functions and accurate functions and give a new score function.

\subsection{Score functions, accurate functions and their limitation analysis}

The existing score functions and accurate functions have specific definitions, but they have limitations for some data. We have carefully combed and explored the existing references and obtained the following seven score functions and accurate functions. After a rigorous review and analysis of the existing references, we believe that the sort function is a means to compare the merits of the two intuitionistic fuzzy numbers, so it is necessary to consider this criterion. In this paper, the limitations of these seven score functions and 
accurate functions are analyzed as follows.

\subsubsection{The $1^{\text {st }}$ kind of score function, accurate function and their limitation analysis}

$\mathrm{Xu}$ [14] defined the score function and accurate function and gave the ordering rules in 2007.

Definition 6 [14] Suppose $\alpha=([a, b],[c, d])$ is an interval-valued intuitionistic fuzzy numbers, its score function $S(\alpha)$ and accurate function $h(\alpha)$ can be defined as follows

$$
S(\alpha)=\frac{(a+b)-(c+d)}{2}, h(\alpha)=\frac{(a+b)+(c+d)}{2} .
$$

The ordering rule: when $S\left(\alpha_{1}\right)>S\left(\alpha_{2}\right)$, the interval-valued intuitionistic fuzzy number $\alpha_{1}$ is better; when $S\left(\alpha_{1}\right)=S\left(\alpha_{2}\right)$, we need to compare the accurate function $h(\alpha)$ (if $h\left(\alpha_{1}\right)>h\left(\alpha_{2}\right)$, the interval-valued intuitionistic fuzzy number $\alpha_{1}$ is better).

Limitation analysis: For some special interval-valued intuitionistic fuzzy numbers satisfying $a_{1}+b_{1}=a_{2}+b_{2}, c_{1}+d_{1}=c_{2}+d_{2}$, this method will fail. For example, we let $\alpha_{1}=([0.35,0.45],[0.20,0.30]), \alpha_{2}=([0.30,0.50],[0.15,0.35]) \quad, \quad$ we can calculate out $S\left(\alpha_{1}\right)=S\left(\alpha_{2}\right)=0.15, h\left(\alpha_{1}\right)=h\left(\alpha_{2}\right)=0.65$. The above results only show $\alpha_{1}$ is equivalent $\alpha_{2}$. We can't judge whether $\alpha_{1}$ or $\alpha_{2}$ is better.

\subsubsection{The $2^{\text {nd }}$ kind of accurate function and its limitation analysis}

After pointing out the limitations of $\mathrm{Xu}$ [14], Ye [23] proposed the following accurate function from the perspective of hesitation in 2009.

Definition 7 [23] Suppose $\alpha=([a, b],[c, d])$ is an interval-valued intuitionistic fuzzy number, its accurate function $h(\alpha)$ can be defined as follows:

$$
h(\alpha)=\frac{a-(1-a-c)+b-(1-b-d)}{2}=a+b-1+\frac{c+d}{2} .
$$

\section{Limitation analysis:}

(1) Let $\alpha_{1}=([0.10,0.20],[0.60,0.80]), \alpha_{2}=([0.40,0.50],[0.10,0.20])$, we can obviously get $\alpha_{1} \subset \alpha_{2}$ and $\alpha_{2}$ is significantly superior to the $\alpha_{1}$. By using Formula (58), we have $h\left(\alpha_{1}\right)=0, h\left(\alpha_{2}\right)=-0.0850, h\left(\alpha_{1}\right)>h\left(\alpha_{2}\right)$. This is clearly not practical.

(2) This function does not make full use of the change information of the upper and lower bounds of membership and non-membership. When the midpoint of the membership degree and the non-membership degree of two interval-valued intuitionistic fuzzy numbers are equal, their exact functions will be equal, and this sort method will fail.

\subsubsection{The $3^{\text {rd }}$ kind of accurate function and its limitation analysis}

In 2011, Nayagam and Sivaraman et al. [24, 25] proposed two accurate functions based on $\mathrm{Xu}$ [14] and Ye [23].

Definition $8[24,25]$ Suppose $\alpha=([a, b],[c, d])$ is an interval-valued intuitionistic fuzzy number, its accurate function $h(\alpha)$ can be defined as follows, where $\delta$ is the weight determined by the decision maker: 


$$
\begin{aligned}
& h(\alpha)=\frac{a+b-d(1-b)-c(1-a)}{2}, \\
& h(\alpha)=\frac{a+b+\delta(2-a-b-c-d)}{2} .
\end{aligned}
$$

\section{Limitation analysis:}

(1) For the first accurate function, $\alpha_{1}=([0.10,0.10],[0.50,0.70]), \alpha_{2}=([0.20,0.20],[0.80,0.80])$, the hesitancy of the latter is $([0.00,0.00],[0.00,0.00])$. Since accurate function places more emphasis on hesitancy and the hesitancy of $\alpha_{2}$ is smaller, we can judge $\alpha_{1}<\alpha_{2}$. However, according to this definition, $h\left(\alpha_{1}\right)=h\left(\alpha_{2}\right)=-0.44$. This is not consistent with common sense.

(2) For the second accurate function, the presence of $\delta$ strengthens the subjective evaluation of the decision maker, and the determination of its value is also a problem.

(3) For two accurate functions, when $a, b, d$ are fixed, we seek the partial derivatives for $c$, then we can find the partial derivative is less than 0 , indicating that the accurate function value increases as the lower bound $c$ of the non-membership interval-value decreases. It is obviously questionable.

\subsubsection{The $4^{\text {th }}$ kind of accurate function and its limitation analysis}

In 2014, Gao et al. [26] proposed the following accurate functions.

Definition 9 [26] Suppose $\alpha=([a, b],[c, d])$ is an interval-valued intuitionistic fuzzy number, its accurate function $h(\alpha)$ can be defined as follows.

$$
h(\alpha)=\frac{a+b-c-d}{(1-b-d)+(1-a-c)+2}=\frac{a+b-c-d}{4-(a+b+c+d)}
$$

Limitation analysis: The accurate function does not make full use of the change information of the upper and lower bounds of the non-membership, when the membership is the same midpoint of the non-membership, the accurate function will be the same, and the sort method of the interval-valued intuitionistic fuzzy number will fail. For example, we let $\alpha_{1}=([0.35,0.45],[0.20,0.30]), \alpha_{2}=([0.30,0.50],[0.15,0.35])$. We have $h\left(\alpha_{1}\right)=h\left(\alpha_{2}\right)=0$. The $\alpha_{1}$ and $\alpha_{2}$ can not be judged in this case.

\subsubsection{The $5^{\text {th }}$ kind of accurate function and its limitation analysis}

In 2015, Kang et al. [27] modified the accurate function and gave the following three definitions.

Definition 10 [27] Suppose $\alpha=([a, b],[c, d])$ is an interval-valued intuitionistic fuzzy number, its accurate function $h(\alpha)$ can be defined as follows, where $\delta_{1}$ and $\delta_{2}$ are weights determined by the intention of the decision maker.

$$
\begin{gathered}
h(\alpha)=\frac{a-c+b-d-c(1-b-d)-d(1-a-c)}{2}, \\
h(\alpha)=\frac{a+b(1-a-c)+b+a(1-b-d)}{2}, \\
h(\alpha)=\frac{a+\delta_{1}(1-a-c)+b+\delta_{2}(1-b-d)}{2} .
\end{gathered}
$$

\section{Limitation analysis:}

(1) When $a, b, d$ are fixed, we seek the partial derivatives for $c$, then we can find the partial derivative is less than 0 , indicating that the accurate function value increases as the lower bound $c$ of the 
non-membership interval-value decreases. which is obviously questionable.

(2) For the three accurate functions, the $\delta$ strengthens the subjective evaluation of the decision maker, and the determination of its value is also a problem.

(3) For Formula (62), let $\alpha_{1}=([0.1,0.5],[0.00,0.00]), \alpha_{2}=([0.20,0.40],[0.00,0.00])$, $h\left(\alpha_{1}\right)=h\left(\alpha_{2}\right)=0.30$. Then $\alpha_{1}$ and $\alpha_{2}$ can not be judged in this case.

(4) For Formula (63), let $\alpha_{1}=([0.00,0.00],[1.00,1.00]), \alpha_{2}=([0.00,0.00],[0.00,0.00]), h\left(\alpha_{1}\right)=h\left(\alpha_{2}\right)=0$. Then $\alpha_{1}$ and $\alpha_{2}$ can not be judged in this case.

\subsubsection{The $6^{\text {th }}$ kind of accurate function and its limitations}

New score functions were presented by Wang et al. [28] in 2017.

Definition 11 [28] Suppose $\alpha=([a, b],[c, d])$ is an interval-valued intuitionistic fuzzy number, its score function $S(\alpha)$ can be defined as follows:

$$
S(\alpha)=\frac{a+b+\sqrt{b d}(1-a-c)+\sqrt{a c}(1-b-d)}{2} .
$$

Limitation analysis: For some special interval-valued intuitionistic fuzzy numbers, this method will fail. For example, let $\alpha_{1}=([0.00,0.00],[0.20,0.30]), \alpha_{2}=([0.00,0.00],[0.10,0.20])$, we have $S\left(\alpha_{1}\right)=S\left(\alpha_{2}\right)=0$, then $\alpha_{1}$ and $\alpha_{2}$ can not be judged in this case.

\subsubsection{The $7^{\text {th }}$ kind of score function and accurate function and their limitations}

In 2018, Wang et al. [29] proposed new score and accurate functions.

Definition 12 [29] Suppose $\alpha=([a, b],[c, d])$ is an interval-valued intuitionistic fuzzy number, its score function $S(\alpha)$ and accurate function $h(\alpha)$ can be defined as follows:

$$
S(\alpha)=\frac{(a+b)(a+c)-(c+d)(b+d)}{2}, h(\alpha)=\frac{(1-a+b)(1-a-c)+(1-c+d)(1-b-d)}{2} .
$$

\section{Limitation analysis:}

For some special interval-valued intuitionistic fuzzy numbers, this method will fail. For example, let $\alpha_{1}=\left(\left[0, b_{1}\right],[0,0]\right), \alpha_{2}=\left(\left[0, b_{2}\right],[0,0]\right)$, we have $S\left(\alpha_{1}\right)=S\left(\alpha_{2}\right)=0, \quad h\left(\alpha_{1}\right)=h\left(\alpha_{2}\right)=1 \quad$ for any $b_{1}, b_{2} \in[0,1]$.

\subsection{Our new score function, theorems and proofs}

In the above cases that the sorting function above has the possibility of failure, we give the new score function drawn from the score function of intuitionistic fuzzy sets of Li [6]. Then the proof of relevant theorems is given.

Definition 16 Suppose $\alpha=([a, b],[c, d])$ is an interval-valued intuitionistic fuzzy number, its score function $S(\alpha)$ can be defined as follows:

$$
S(\alpha)=(a+b-1)+\frac{2-a-b-c-d}{2+c+d} .
$$

The formula considers membership, non-membership and hesitancy of the interval-valued intuitionistic fuzzy set. Let 88 


$$
\psi_{A}=\frac{a+b}{2}, \Delta_{A}=\frac{c+d}{2},
$$

then this formula satisfies the following theorems.

Theorem 3 Sore function $S(\alpha)$ is monotonically increasing about $\psi_{A}$ and is monotonically decreasing about $\Delta_{A}$.

Proof:

Since

$$
\begin{gathered}
S(\alpha)=(a+b-1)+\frac{2-a-b-c-d}{2+c+d}=2 \psi_{A}-1+\frac{1-\psi_{A}-\Delta_{A}}{1+\Delta_{A}}, \\
\frac{\partial S}{\partial \psi_{A}}=2-\frac{1}{1+\Delta_{A}}>0, \\
\frac{\partial S}{\partial \Delta_{A}}=\frac{-2+\psi_{A}}{\left(1+\Delta_{A}\right)^{2}}<0,
\end{gathered}
$$

we can get score function $S(\alpha)$ is monotonically increasing about $\psi_{A}$ and is monotonically decreasing about $\Delta_{A}$.

Theorem 4 Score function $S(\alpha)$ is bounded and $S(\alpha) \in[-1,1]$.

\section{Proof:}

According to Theorem 3, S( $\alpha)$ is monotonically increasing about $\psi_{A}$ and is monotonically decreasing about $\Delta_{A}$. So if and only if $\psi_{A}=1, \Delta_{A}=0, S(\alpha)$ has the maximum value of 1 ; if and only if $\psi_{A}=0, \Delta_{A}=1, S(\alpha)$ has the minimum value of -1 . So $S(\alpha) \in[-1,1]$, i.e. $S(\alpha)$ is bounded.

Theorem 5 Suppose $\alpha_{1}=\left(\left[a_{1}, b_{1}\right],\left[c_{1}, d_{1}\right]\right)$ and $\alpha_{2}=\left(\left[a_{2}, b_{2}\right],\left[c_{2}, d_{2}\right]\right)$ are both interval-valued intuitionistic fuzzy numbers, when $\alpha_{1} \supseteq \alpha_{2}, S\left(\alpha_{1}\right) \geq S\left(\alpha_{2}\right)$, when $\alpha_{1} \subseteq \alpha_{2}, S\left(\alpha_{1}\right) \leq S\left(\alpha_{2}\right)$.

\section{Proof:}

When

$$
\alpha_{1} \supseteq \alpha_{2}, \quad a_{1} \geq a_{2}, b_{1} \geq b_{2}, c_{1} \leq c_{2}, d_{1} \leq d_{2},
$$

we have

$$
\psi_{\alpha_{1}}=\frac{a_{1}+b_{1}}{2} \geq \psi_{\alpha_{2}}=\frac{a_{2}+b_{2}}{2}, \Delta_{\alpha_{1}}=\frac{c_{1}+d_{1}}{2} \leq \Delta_{\alpha_{2}}=\frac{c_{2}+d_{2}}{2},
$$

According to Theorem 3, we have

$$
S\left(\alpha_{1}\right) \geq S\left(\alpha_{2}\right)
$$

Similarly, we also can prove $S\left(\alpha_{1}\right) \leq S\left(\alpha_{2}\right)$ when $\alpha_{1} \subseteq \alpha_{2}$.

Remark 5 The score function proposed by Li (2015) is based on the intuitionistic fuzzy set. Here we extend it to the interval-valued intuitionistic fuzzy set. The original score function is

$$
S(\alpha)=\left(2 \mu_{\alpha}-1\right)+\frac{\pi_{\alpha}}{1+v_{\alpha}} .
$$

Where $\alpha=\left(\mu_{\alpha}, v_{\alpha}\right), \mu_{\alpha}$ represents the membership of $\alpha, v_{\alpha}$ is the non-membership of $\alpha$ and $\pi_{\alpha}$ is the hesitating degree of $\alpha$. When $\alpha=([a, b],[c, d])$, we let 


$$
\mu_{\alpha}=\frac{a+b}{2}, v_{\alpha}=\frac{c+d}{2}, \pi_{\alpha}=1-\left(\frac{a+b}{2}+\frac{c+d}{2}\right)=\frac{2-a-b-c-d}{2} .
$$

Finally, we obtain the score function defined above

$$
S(\alpha)=(a+b-1)+\frac{2-a-b-c-d}{2+c+d} .
$$

\subsection{Comparison of our new score function with existing sort functions}

We get the following table by comparing the existing score and accurate functions with the new score function. The results show that the merits of certain interval-valued intuitionistic fuzzy numbers can not be judged through the existing sort functions, while the new sort function can obtain reasonable sorting results.

\begin{tabular}{|c|c|c|c|c|c|}
\hline Formula & Reference & Examples & $\begin{array}{c}\text { Results of } \\
\text { the sort }\end{array}$ & Remark & $\begin{array}{c}\text { Our new } \\
\text { function (67) }\end{array}$ \\
\hline (57) & $\begin{array}{l}\text { reference [14] } \\
(\mathrm{Xu} 2007)\end{array}$ & $\begin{array}{l}\alpha_{1}=([0.35,0.45],[0.20,0.30]) \\
\alpha_{2}=([0.30,0.50],[0.15,0.35])\end{array}$ & $\alpha_{1}=\alpha_{2}$ & $\begin{array}{l}\text { Unable to } \\
\text { judge }\end{array}$ & $\alpha_{1}<\alpha_{2}$ \\
\hline (58) & $\begin{array}{l}\text { reference [23] } \\
\quad(\text { Ye 2009) }\end{array}$ & $\begin{array}{l}\alpha_{1}=([0.10,0.20],[0.60,0.80]) \\
\alpha_{2}=([0.40,0.50],[0.01,0.02])\end{array}$ & $\alpha_{1}>\alpha_{2}$ & $\begin{array}{l}\text { In fact, } \\
\alpha_{1}<\alpha_{2}\end{array}$ & $\alpha_{1}<\alpha_{2}$ \\
\hline (59) & $\begin{array}{c}\text { reference [24] } \\
\text { (Nayagam 2011) }\end{array}$ & $\begin{array}{l}\alpha_{1}=([0.10,0.10],[0.50,0.70]) \\
\alpha_{2}=([0.20,0.20],[0.80,0.80])\end{array}$ & $\alpha_{1}=\alpha_{2}$ & $\begin{array}{l}\text { Unable to } \\
\text { judge }\end{array}$ & $\alpha_{1}<\alpha_{2}$ \\
\hline (61) & $\begin{array}{l}\text { reference [26] } \\
(\text { Gao 2014) }\end{array}$ & $\begin{array}{l}\alpha_{1}=([0.35,0.45],[0.20,0.30]) \\
\alpha_{2}=([0.30,0.50],[0.15,0.35])\end{array}$ & $\alpha_{1}=\alpha_{2}$ & $\begin{array}{l}\text { Unable to } \\
\text { judge }\end{array}$ & $\alpha_{1}<\alpha_{2}$ \\
\hline (62) & $\begin{array}{l}\text { reference [27] } \\
\text { (Kang 2015) }\end{array}$ & $\begin{array}{l}\alpha_{1}=([0.10,0.50],[0.00,0.00]) \\
\alpha_{2}=([0.20,0.40],[0.00,0.00])\end{array}$ & $\alpha_{1}=\alpha_{2}$ & $\begin{array}{l}\text { Unable to } \\
\text { judge }\end{array}$ & $\alpha_{1}>\alpha_{2}$ \\
\hline$(65)$ & $\begin{array}{l}\text { reference [28] } \\
\text { (Wang 2017) }\end{array}$ & $\begin{array}{l}\alpha_{1}=([0.00,0.50],[0.00,0.00]) \\
\alpha_{2}=([0.00,0.60],[0.00,0.00])\end{array}$ & $\alpha_{1}=\alpha_{2}$ & $\begin{array}{l}\text { Unable to } \\
\text { judge }\end{array}$ & $\alpha_{1}<\alpha_{2}$ \\
\hline (66) & $\begin{array}{l}\text { reference [29] } \\
\text { (Wang 2018) }\end{array}$ & $\begin{array}{l}\alpha_{1}=([0.00,0.00],[0.00,0.00]) \\
\alpha_{2}=([0.00,1.00],[0.00,0.00])\end{array}$ & $\alpha_{1}=\alpha_{2}$ & $\begin{array}{l}\text { In fact, } \\
\alpha_{1}<\alpha_{2}\end{array}$ & $\alpha_{1}<\alpha_{2}$ \\
\hline
\end{tabular}

Table 2: Comparison of our new score function with existing sort functions

(1) For $\alpha_{1}=\overline{([0.35,0.45],[0.20,0.30])}$ and $\alpha_{2}=([0.30,0.50],[0.15,0.35])$, we have $\alpha_{1}=\alpha_{2}$ according to the function in reference [14], then we can't judge which is better. In fact, if two interval-valued intuitionistic fuzzy numbers satisfying $a_{1}+b_{1}=a_{2}+b_{2}$ and $c_{1}+d_{1}=c_{2}+d_{2}$, this function will fail. But we can obtain $\alpha_{1}<\alpha_{2}$ by our new function.

(2) For $\alpha_{1}=([0.10,0.20],[0.60,0.80])$ and $\alpha_{2}=([0.40,0.50],[0.01,0.02])$, we can obviously get $\alpha_{1} \subset \alpha_{2}$ and $\alpha_{2}$ is significantly superior to the $\alpha_{1}$. But according to reference [23], we have $\alpha_{1}=\alpha_{2}$ which is not practical. Then we obtain the reasonable result $\alpha_{1}<\alpha_{2}$ by our new function. Furthermore, if two interval-valued intuitionistic fuzzy numbers satisfying $a_{1}+b_{1}=a_{2}+b_{2}$ and $c_{1}+d_{1}=c_{2}+d_{2}$, the sort function in reference [23] also can't judge which is better. But our new function can handle this situation effectively.

(3) For $\alpha_{1}=([0.10,0.10],[0.50,0.70])$ and $\alpha_{2}=([0.20,0.20],[0.80,0.80])$, the hesitancy of the latter is ([0.00], [0.00]). Intuitively, we can judge $\alpha_{1}<\alpha_{2}$. However, according to this function in reference [24], 
we have $\alpha_{1}=\alpha_{2}$ which is not consistent with common sense. The result of our new function is $\alpha_{1}<\alpha_{2}$ which is reasonable.

(4) For $\alpha_{1}=([0.35,0.45],[0.20,0.30])$ and $\alpha_{2}=([0.30,0.50],[0.15,0.35])$. We have $\alpha_{1}=\alpha_{2}$ according to the function in reference [26]. Then $\alpha_{1}$ and $\alpha_{2}$ can't be judged in this case. In fact, the function does not make full use of the information of the upper and lower bounds of the non-membership which means if two interval-valued intuitionistic fuzzy numbers satisfying $c_{1}+d_{1}=c_{2}+d_{2}$, the two function values will be equal and the function will fail. We can obtain $\alpha_{1}<\alpha_{2}$ by our new function, it means that our new function can make full use of the information of the upper and lower bounds of the non-membership.

(5) For $\alpha_{1}=([0.10,0.50],[0.00,0.00])$ and $\alpha_{2}=([0.20,0.40],[0.00,0.00])$, we can't judge which is better according to the function in reference [27]. In fact, if two interval-valued intuitionistic fuzzy numbers satisfying $a_{1}+b_{1}=a_{2}+b_{2}$ and $c_{1}+d_{1}=c_{2}+d_{2}$, this function will fail. But we have $\alpha_{1}<\alpha_{2}$ according to our new function.

(6) For $\alpha_{1}=([0.00,0.00],[0.20,0.30]) \quad$ and $\quad \alpha_{2}=([0.00,0.00],[0.10,0.20])$, we have $S\left(\alpha_{1}\right)=S\left(\alpha_{2}\right)=0$ according to the function in reference [28] and we can't judge which is better. In fact, if $a=b=0$, then we will have $S(\alpha)=0$. But our new function does not be influenced by this case and we can obtain $\alpha_{1}<\alpha_{2}$.

(7) For $\alpha_{1}=([0.00,0.00],[0.00,0.00])$ and $\alpha_{2}=([0.00,1.00],[0.00,0.00])$, we have $\alpha_{1}=\alpha_{2}$ according to sort functions in reference [29]. In this case, we can't judge which is better. In fact, if $\alpha_{1}=\left(\left[0.00, b_{1}\right],[0.00,0.00]\right) \quad$ and $\quad \alpha_{2}=\left(\left[0.00, b_{2}\right],[0.00,0.00]\right) \quad$, we have $S\left(\alpha_{1}\right)=S\left(\alpha_{2}\right)=0, h\left(\alpha_{1}\right)=h\left(\alpha_{2}\right)=1$ for any $b_{1}, b_{2} \in[0,1]$. But we can judge which is better according to our new function if $b_{1} \neq b_{2}$.

\section{A novel fuzzy portfolio model with improved interval-valued intuitionistic AHP and score function}

Based on the interval-valued intuitionistic fuzzy hierarchical analysis method, we construct a novel fuzzy portfolio model with improved interval-valued intuitionistic AHP and new score function and give a numerical example to verify the feasibility and effectiveness of our proposed model and methods.

\subsection{Model building}

Suppose $A=\left\{A_{1}, A_{2}, \ldots, A_{n}\right\}$ is a portfolio collection of interval-valued intuitionistic fuzzy portfolio model problems, $C=\left\{C_{1}, C_{2}, \ldots, C_{m}\right\} \quad$ is an attribute set, $\omega=\left\{\omega_{1}, \omega_{2}, \ldots, \omega_{m}\right\}$ is the weight vector of the attribute, $\omega_{j}$ represents the weight of $C_{j}$ satisfying $\sum_{j=1}^{m} \omega_{j}=1$, where the weight is unknown. The decision maker measure portfolio $A_{i}(i=1,2, \ldots, n)$ about the attribute $C_{j}(i=1,2, \ldots, m)$ and give interval-valued intuitionistic fuzzy number $r_{i j}=\left(\left[a_{i j}, b_{i j}\right],\left[c_{i j}, d_{i j}\right]\right),\left[a_{i j}, b_{i j}\right]$ represents the degree to which the decision maker is satisfied with the portfolio $A_{i}$ with respect to attribute $C_{j},\left[c_{i j}, d_{i j}\right]$ represents the degree to which the decision maker is not satisfied with the portfolio $A_{i}$ with respect to attribute $C_{j}$, then combine $r_{i j}=\left(\left[a_{i j}, b_{i j}\right],\left[c_{i j}, d_{i j}\right]\right)$ and form the decision matrix $R=\left(r_{i j}\right)_{m \times n}, i=1,2, \ldots, n, j=1,2, \ldots, m$. 
To solve these problems, we propose a multi-attribute decision making method based on the improved interval-valued intuitionistic fuzzy AHP and new score function. Its specific steps are as follows.

Step 1: According to the interval-valued intuitionistic fuzzy judgment matrix $R^{\prime}=\left(r_{i j}\right)_{n \times m}$, we can calculate the weight vector $\omega^{\prime}=\left\{\omega_{1}{ }^{\prime}, \omega_{2}{ }^{\prime}, \ldots, \omega_{m}{ }^{\prime}\right\}$ of attribute index by Formulae (50)-(56).

Step 2: According to the decision matrix $R$, we can calculate the weight vector $\omega^{*}=\left\{\omega_{1}^{*}, \omega_{2}^{*}, \ldots, \omega_{m}^{*}\right\}$ of the attribute by

$$
\begin{gathered}
e_{j}=\frac{1}{n} \sum_{i=1}^{n} \frac{2-\left|\mu_{i j}^{L}(x)-v_{i j}^{L}(x)\right|-\left|\mu_{i j}^{U}(x)-v_{i j}^{U}(x)\right|+\pi_{i j}^{U}(x)+\pi_{i j}^{U}(x)}{2+\left|\mu_{i j}^{L}(x)-v_{i j}^{L}(x)\right|+\left|\mu_{i j}^{U}(x)-v_{i j}^{U}(x)\right|+\pi_{i j}^{U}(x)+\pi_{i j}^{U}(x)}, \\
\omega_{j}^{*}=\frac{1-e_{j}}{m-\sum_{j=1}^{m} e_{j}} .
\end{gathered}
$$

Step 3: From $\omega^{\prime}=\left\{\omega_{1}{ }^{\prime}, \omega_{2}{ }^{\prime}, \ldots, \omega_{m}{ }^{\prime}\right\}$ and $\omega^{*}=\left\{\omega_{1}^{*}, \omega_{2}^{*}, \ldots, \omega_{m}^{*}\right\}$, we can obtain the combination weight vector $\omega=\left\{\omega_{1}, \omega_{2}, \ldots, \omega_{m}\right\}$ of the attribute index by

$$
\omega_{i}=k \omega_{i}^{\prime}+(1-k) \omega_{i}^{*}
$$

Step 4: Calculate the combination attribute values of $A_{i}$

$$
r_{i}=\left(\left[a_{i}, b_{i}\right],\left[c_{i}, d_{i}\right]\right)=\sum_{j=1}^{m} \omega_{i j} \alpha_{i j}=\left(\left[1-\prod_{j=1}^{m}\left(1-a_{i j}\right)^{\omega_{i j}}, 1-\prod_{j=1}^{m}\left(1-b_{i j}\right)^{\omega_{i j}}\right],\left[\prod_{j=1}^{m} c_{i j}^{\omega_{i j}}, \prod_{j=1}^{m} d_{i j}^{\omega_{i j}}\right]\right) .
$$

Step 5: Calculate the score function value $S\left(A_{i}\right)$ of $A_{i}$ by using Formula (67).

Step 6: We sort the alternative portfolios according to the score function value $S\left(A_{i}\right)$.

Schematic diagram of the entropy method-interval-valued intuitionistic fuzzy AHP are as follows.

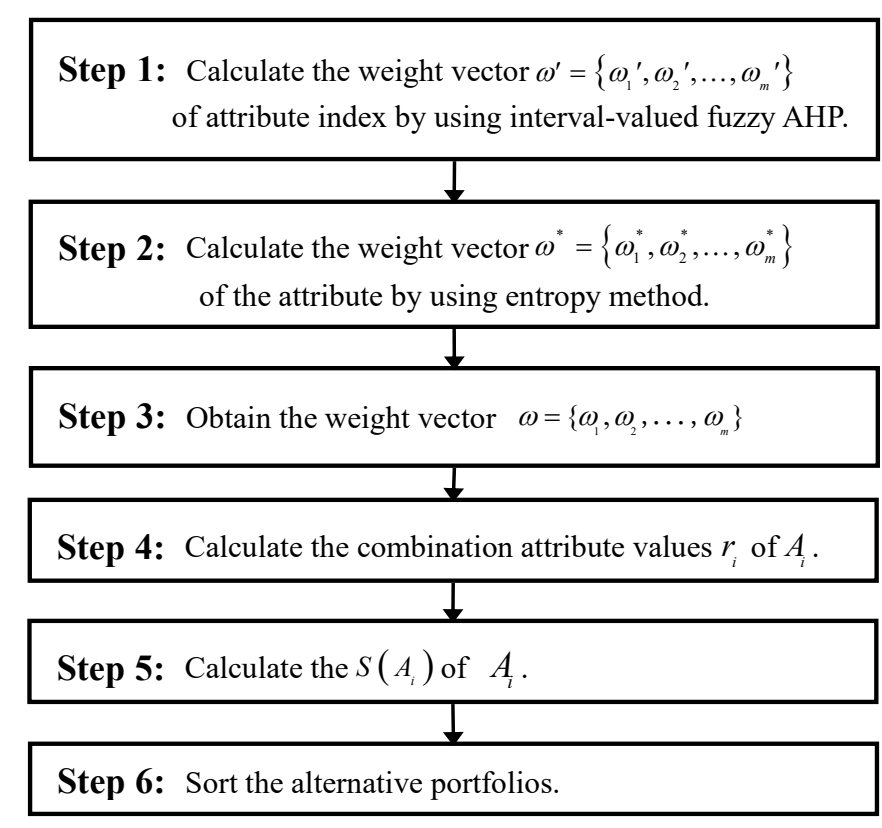

Figure 2: Schematic diagram of the entropy method-interval-valued intuitionistic fuzzy AHP.

\subsection{Numerical example}

To better choose the portfolio, we usually evaluate the portfolio with income, risk, Sharp ratio, and this paper uses income, risk, Sharp ratio as attributes, then we consider an investor who wants to buy a portfolio. There are two kinds of portfolios $A=\left\{A_{1}, A_{2}\right\}$ and three attributes which are rate of return $C_{1}$, risk $C_{2}$ 
and Sharpe ratio $C_{3}$. After the data processing, we can obtain the interval-valued intuitionistic fuzzy judgment matrix $R^{\prime}=\left(r_{i j}{ }^{\prime}\right)_{n \times m}$ as shown in Table 3 .

Table 3: Judgment matrix $R^{\prime}=\left(r_{i j}{ }^{\prime}\right)_{n \times m}$

\begin{tabular}{cccc}
\hline Attribute & $C_{1}$ & $C_{2}$ & $C_{3}$ \\
\hline$C_{1}$ & $([0.50,0.50],[0.50,0.50])$ & $([0.60,0.70],[0.15,0.30])$ & $([0.55,0.65],[0.20,0.35])$ \\
$C_{2}$ & $([0.15,0.30],[0.60,0.70])$ & $([0.50,0.50],[0.50,0.50])$ & $([0.65,0.75],[0.10,0.25])$ \\
$C_{3}$ & $([0.20,0.35],[0.55,0.65])$ & $([0.10,0.25],[0.65,0.75])$ & $([0.50,0.50],[0.50,0.50])$ \\
\hline
\end{tabular}

After data processing, we can obtain the interval-valued intuitionistic fuzzy decision matrix as shown in Table 4.

Table 4: Decision matrix

\begin{tabular}{cccc}
\hline Portfolio & $C_{1}$ & $C_{2}$ & $C_{3}$ \\
\hline$A_{1}$ & $([0.60,0.70],[0.20,0.30])$ & $([0.40,0.50],[0.20,0.40])$ & $([0.70,0.80],[0.10,0.20])$ \\
$A_{2}$ & $([0.50,0.60],[0.10,0.30])$ & $([0.60,0.70],[0.20,0.30])$ & $([0.60,0.70],[0.10,0.20])$ \\
\hline
\end{tabular}

Step 1: According to the judgment matrix $R^{\prime}=\left(r_{i j}{ }^{\prime}\right)_{n \times m}$, we can calculate the weight vector of the attribute index by using Formulae (50)-(56). ( $\left.\lambda_{i j}=0.5\right)$. It is $\omega^{\prime}=\{0.4121,0.3435,0.2444\}$.

Step 2: We use Formulae (78) and (79) to calculate the weight vector as shown below:

$$
\omega^{*}=\{0.3222,0.2482,0.4296\} .
$$

Step 3: From $\omega^{\prime}=\{0.4121,0.3435,0.2444\}$ and $\omega^{*}=\{0.3222,0.2482,0.4296\}$, we use Formula $(80)(k=0.5)$ to obtain the combination weighting vector as $\omega=\{0.36715,0.29585,0.337\}$.

Step 4: We use Formula (81) to calculate the combination attribute values of portfolio $A_{i}(i=1,2)$ as:

$$
\begin{aligned}
& r_{1}=([0.5907,0.6956],[0.1583,0.2849]), \\
& r_{2}=([0.5658,0.6666],[0.1228,0.2617]) .
\end{aligned}
$$

Step 5: We can calculate the score function value of combination attribute value $S\left(A_{i}\right)$ of $A_{i}$ by using Formula (68)

$$
S\left(A_{1}\right)=0.3970, S\left(A_{2}\right)=0.3931 .
$$

Step 6: We sort the alternative portfolios according to the score function value $S\left(A_{i}\right)$. We can get that $A_{1} \succ A_{2}$, so the portfolio $A_{1}$ is selected:

$$
S\left(A_{1}\right)>S\left(A_{2}\right) .
$$

Through calculation, the sort function value of each portfolio is obtained. The sorting results are finally given, as shown in Table 5.

Table 5: Sorting results of different portfolios

\begin{tabular}{cccccc}
\hline \multirow{2}{*}{ Formulae } & \multicolumn{2}{c}{ Original score } & \multicolumn{3}{c}{ Normalized score } \\
& $A_{1}$ & $A_{2}$ & $A_{1}$ & $A_{2}$ & Sorting results \\
\hline$(57)$ & 0.4216 & 0.4240 & 0.4986 & 0.5014 & $A_{1} \prec A_{2}$ \\
$(58)$ & 0.5079 & 0.4246 & 0.5447 & 0.4553 & $A_{1} \succ A_{2}$ \\
$(59)$ & 1.1350 & 1.0920 & 0.5097 & 0.4903 & $A_{1} \succ A_{2}$ \\
\hline
\end{tabular}




\begin{tabular}{cccccc}
\hline$(61)$ & 0.3713 & 0.3558 & 0.5107 & 0.4893 & $A_{1} \succ A_{2}$ \\
$(62)$ & 0.3843 & 0.3788 & 0.5036 & 0.4964 & $A_{1} \succ A_{2}$ \\
$(65)$ & 1.4040 & 1.3810 & 0.5041 & 0.4959 & $A_{1} \succ A_{2}$ \\
$(66)$ & 0.2644 & 0.2458 & 0.5182 & 0.4818 & $A_{1} \succ A_{2}$ \\
Our new function (67) & $\mathbf{0 . 3 9 7 0}$ & $\mathbf{0 . 3 9 3 1}$ & $\mathbf{0 . 5 0 2 5}$ & $\mathbf{0 . 4 9 7 5}$ & $A_{1} \succ A_{2}$ \\
\hline
\end{tabular}

From the above table, we find that under six formulae, $A_{1}$ is the optimal portfolio. What we find is that the new score function has the same sorting result as these six formulae. In order to more intuitively represent the sorting result obtained by the existing sort functions, the charts of original score of each portfolio is given below, as shown in Figure 3. We draw bar charts and line charts of the original score for two portfolios. From these bar charts, we can see the original sores of the two portfolios obtained by each sort function. Only the original sore of $A_{1}$ obtained by Formula (57) is larger than that of $A_{2}$ $(0.4240>0.4216)$. The original sores of $A_{1}$ obtained by the other six formulae are larger than that of $A_{2}$. Our new score function achieves the same result with these six formulae. From these line charts, we can find that The line chart for portfolio $A_{1}$ is basically above the line chart for portfolio $A_{2}$ (except for Formula (57)), which intuitively shows that portfolio $A_{1}$ is superior to portfolio $A_{2}$.

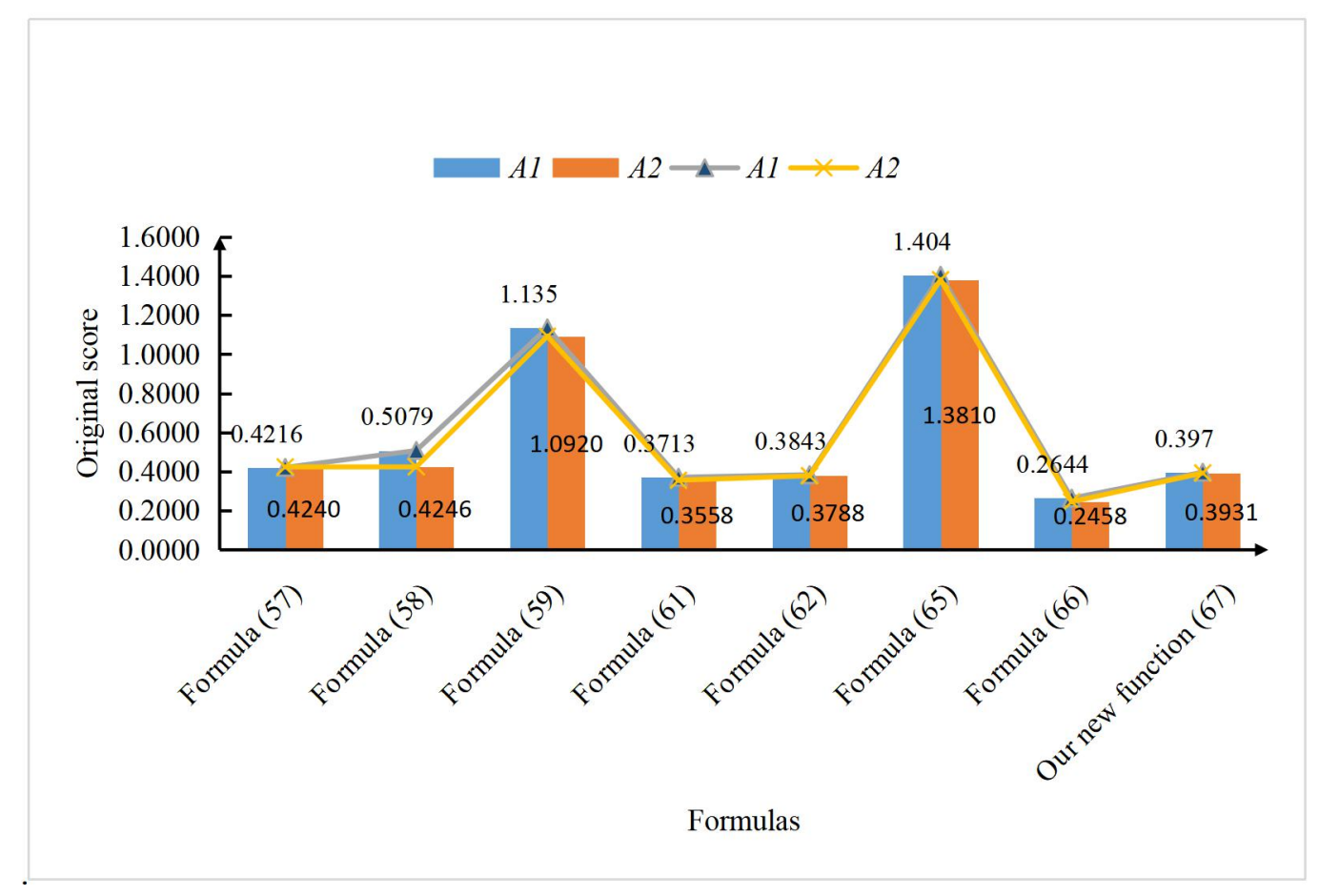

Figure 3: Original score of different portfolios

In order to eliminate the influence of dimension on the result of function values, we normalized the function values obtained by each formula. The charts of normalized score of different portfolios is given below, as shown in Figure 4. 


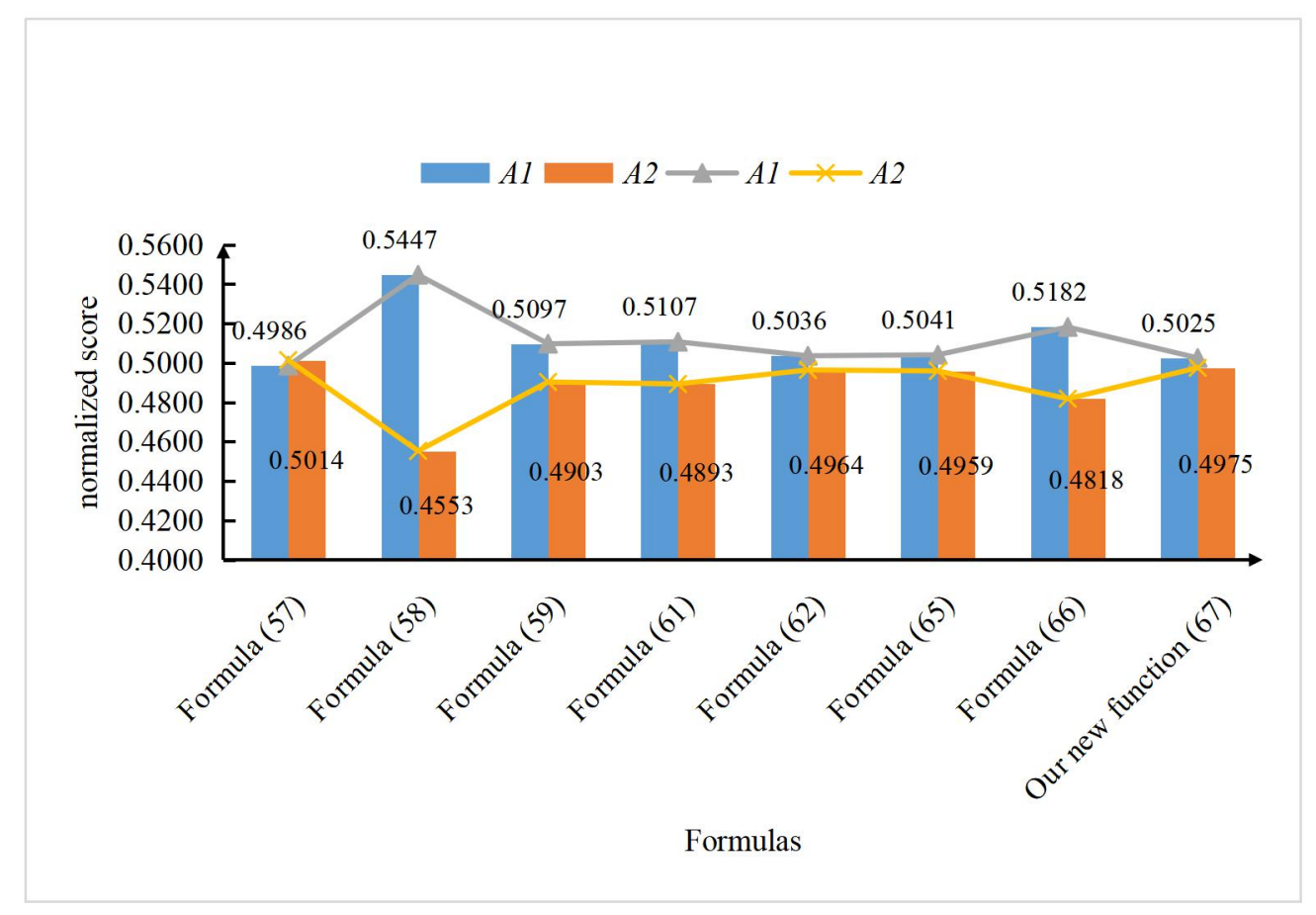

Figure 4: Normalized score of different portfolios

We draw bar charts and line charts of the normalized scores for two portfolios. From these bar charts, we can see the normalized sores of the two portfolios obtained by each sort function. Only the normalized sore of $A_{1}$ obtained by Formula (57) is larger than that of $A_{2} \quad(0.5014>0.4986)$. The normalized sores of $A_{1}$ obtained by the other six formulae are larger than that of $A_{2}$. Our new score function achieves the same result with these six formulae. From these line charts, we can find that the normalized sore of $A_{1}$ is basically bigger than 0.5 and the normalized sore of $A_{2}$ is basically smaller than 0.5 (except for Formula (57)), which intuitively shows that portfolio $A_{1}$ is superior to portfolio $A_{2}$.

From the figures above, we can see the sorting results obtained by the existing 7 sort functions. Of these, the better is $A_{1}$. And the sorting result we get with the new score function is $A_{1} \succ A_{2}$. Combined with the analysis in Section4, it is doubtless that the result of the new score function is better in application.

\section{Conclusion}

With the help of Xu [21]'s intuitionistic fuzzy AHP, we apply AHP to the context of interval-valued intuitionistic fuzzy sets. Because the interval-valued intuitionistic fuzzy set is powerful in describing fuzziness and uncertainty, the interval-valued intuitionistic fuzzy AHP can describe the decision-making process more accurately, which makes the interval-valued intuitionistic fuzzy AHP have more advantages than AHP and FAHP. It can solve the problem that the classical AHP ignores the fuzziness of expert judgment. In addition, we combine interval-valued intuitionistic fuzzy AHP and entropy weight method to get the combined weight method, which overcomes the limitations of unilateral authorization method. By the score function obtained by adjusting the parameters, we solve the problem that the previous sort functions are invalid for some interval intuitionistic fuzzy numbers. Finally, we propose a novel portfolio with the improved intuitionistic AHP and the new score function. The feasibility and validity of the model are proved by applying it to the portfolio decision problem through an example. These result of our proposed new score function is consistent with those of most existing sort functions. Therefore, our proposed score function is not only effective in practice, but also able to overcome some shortcomings of 
other score functions in theory. The research results of this paper will provide more theoretical and practical reference for investment decision makers. In the future research, we are interested in further theoretical discussion of entropy weight method and the application of new analytic hierarchy process combined with dual hesitation fuzzy sets.

\section{Author Contributions}

Xue Deng and Jianxin Yang were responsible for the overall understanding of the structure of the article, the main idea of the article, the conclusion analysis of the article and the revision of the full text.

Fengting Geng performed the data analyses and wrote the manuscript.

\section{Ethical approval}

This article does not contain any studies with human participants or animals performed by any of the authors.

\section{Acknowledgements}

This research was supported by the "National Social Science Foundation Projects of China, No. 21BTJ069". The authors are highly grateful to Mr. Chuangjie Chen, Dr. Wenhua Gao, the referees and editor in-chief for their very helpful advices and comments.

\section{Conflict of interest}

We declare that we have no conflict of interest.

\section{Informed consent}

Informed consent was obtained from all individual participants included in the study.

\section{References}

[1] H. Markowitz, Portfolio selection, The Journal of Finance, 7 (1952), 77-91.

[2] T.D. Warren, D.B. James, The Mean and Variance of Watson's Distribution, John Wiley \& Sons, 36 (1974).

[3] W.V. Harlow, Asset Allocation in a Downside-Risk Framework, Financial Analysts Journal, 47 (1991)

[4] H. Konno, R.P. Stanley, K. Suzuki, Optimal portfolios with asymptotic criteria, Annals of Operations Research, 45 (1993).

[5] H. Konno, H. Yamazaki, Mean-absolute deviation portfolio optimization model and its applications to Tokyo stock market Management Science, 37 (1991), 519-531.

[6] G. Athayde, R. Flores, Finding a maximum skewness portfolio: A general solution to three-moments portfolio choice, Journal of Economic Dynamics and Control. 28 (2004), 1335 - 1352.

[7] E. Jondeau, M. Rockinger, Testing for differences in the tails of stock-market returns, Empir.Finance 10 (2003), $559-581$.

[8] P. Li, Y.W. Han, Y. Xia, Portfolio optimization using asymmetry robust mean absolute deviation model, Finance Research Letters 18 (2016), 353 - 362.

[9] L. A. Zadeh, Fuzzy sets, Inform and Control, 8 (1965), 338-356.

[10] T.L. Saaty, L.G. Vargas, Estimating technological coefficients by the analytic hierarchy process, Pergamon, 13 (1979).

[11] K.T. Atanassov, Intuitionistic fuzzy sets, Fuzzy Sets \& Systems, 20 (1986), 87-96. 
[12] K.T. Atanassov, G. Gargov, interval-valued valued intuitionistic fuzzy sets, Fuzzy Sets \& Systems, 31 (1989), 343-349.

[13] R. Sadiq, S. Tesfamariam, Environmental decision-making under uncertainty using intuitionistic fuzzy analytic hierarchy process (IF-AHP), Stochastic Environmental Research and Risk Assessment, 23 (2009), 75-91.

[14] Z.S. Xu, Integration method of interval-valued intuitive fuzzy information and its application in decision-making, Control and decision-making, 22 (2007), 215-219.

[15] M. Li, C. Wu, L. Zhang, Intuitionistic Fuzzy Decision Method Based on Prospect Theory and Its Application in Distribution Center Location. Journal of Xihua University (Natural Science Edition), 34 (2015), 1-5+11.

[16] J.J. Wang, Using the fuzzy multi-criteria model to select the optimal cool storage system for air conditioning, Energy \& Buildings, 40 (2008), 2059-2066.

[17] L.Z. Yi, Health Status Sensing of Catenary Based on Combination Weighting and Normal Cloud Model, Arabian Journal for Science and Engineering, (2021), 1-15.

[18] Y.S. Hu, C.L. Zhu, Y. Li, Credit Evaluation Model of Road Transportation Enterprises Based on the Combination Weighting Method, Mathematical Problems in Engineering, (2021).

[19] F. Tan, Suitability evaluation of underground space based on finite interval-valued cloud model and genetic algorithm combination weighting, Tunneling and Underground Space Technology. 108 (2021), 103743-103744.

[20] Z.X. Wu., S. Huang, W.L. Hu, Research on the Selection of Green Building Projects based on Combination Weighting-Improved TOPSIS Method, World Scientific Research Journal, 6 (2020).

[21] Z.S. Xu, H. Liao, Intuitionistic fuzzy analytic hierarchy process. IEEE transactions on fuzzy systems, 22 (2014), 749-761.

[22] R. Verma, S. Chandra, interval-valued intuitionistic fuzzy-analytic hierarchy process for evaluating the impact of security attributes in fog-based Internet of Things paradigm, Computer Communications, (2021)

[23] J. Ye, Multi-criteria fuzzy decision-making method based on a novel accuracy function under interval-valued intuitionistic fuzzy environment, Expert systems with Applications, 36 (2009), 6899-6902.

[24] V.L.G. Nayagam, S. Muralikrishnan, G. Sivaraman, Multi-criteria decision-making method based on interval-valued intuitionistic fuzzy sets, Expert Systems with Applications, 38 (2011), 1464-1467.

[25] V.L.G. Nayagam, G. Sivaraman, Ranking of interval-valued intuitionistic fuzzy sets, Applied Soft Computing, 11 (2011), 3368-3372.

[26] J.W. Gao, H.H. Liu, Y.D. Gu, interval-valued Intuition Fuzzy Multi-Criterion Decision Method Based on Foreground Theory, Theory and practice of system engineering, 34 (2014), 3175-3181.

[27] J. Kang, R. Lan, S.S. Wang, The accurate function of the interval-valued intuitive ambiguity and its application to decision making, Xi'an University of Posts and Telecommunications Journal, 20 (2015), 86-91.

[28] C.Y. Wang, S.M. Chen, An improved multi-attribute decision making method based on new score function of interval-valued intuitionistic fuzzy values and linear programming methodology, Information Sciences, 411 (2017), 176-184.

[29] C.Y. Wang, S.M. Chen, A new multiple attribute decision making method based on linear programming methodology and novel score function and novel accuracy function of interval-valued intuitionistic fuzzy values, Information Sciences, 438 (2018), 145-155.

[30] P. Burillo, H. Bustince, Entropy on intuitionistic fuzzy sets and on interval-valued fuzzy sets, Fuzzy Sets and Systems, 118 (2001), 305-316. 
[31] Szmidt, J. Kacprzyk, Entropy for intuitionistic fuzzy sets, Fuzzy Sets and Systems, 118 (2001), 467-477.

[32] X. Deng, Y.K. Yuan, A novel fuzzy dominant goal programming for portfolio selection with systematic risk and non-systematic risk, Soft Computing, 25 (2021), 14809-14828.

[33] X. Deng, Y.Y. Liu, Analysis on the Development of Digital Economy in Guangdong Province Based on Improved Entropy Method and Multivariate Statistical Analysis, Entropy, 22 (2021). 